\title{
Modal contribution and state space order selection in operational modal analysis
}

\author{
F. Javier Cara ${ }^{\mathrm{a}, *}$, Jesús Juan ${ }^{\mathrm{a}}$, Enrique Alarcón ${ }^{\mathrm{b}}$, \\ Edwin Reynders ${ }^{c}$, Guido De Roeck ${ }^{\mathrm{c}}$
}

${ }^{a}$ Laboratory of Statistics, ETS Ingenieros Industriales, Universidad Politécnica de Madrid. José Gutiérrez Abascal, 2. 28006, Madrid, Spain

${ }^{\mathrm{b}}$ Department of Structural Mechanics, ETS Ingenieros Industriales, Universidad Politécnica de Madrid. José Gutiérrez Abascal,

2. 28006, Madrid, Spain

${ }^{\circ}$ Department of Civil Engineering, K.U. Leuven, Kasteelpark Arenberg 40, B-3001 Leuven, Belgium

\begin{abstract}
A B S T R A C T
The estimation of modal parameters of a structure from ambient measurements has attracted the attention of many researchers in the last years. The procedure is now well established and the use of state space models, stochastic system identification methods and stabilization diagrams allows to identify the modes of the structure. In this paper the contribution of each identified mode to the measured vibration is discussed. This modal contribution is computed using the Kalman filter and it is an indicator of the importance of the modes. Also the variation of the modal contribution with the order of the model is studied. This analysis suggests selecting the order for the state space model as the order that includes the modes with higher contribution. The order obtained using this method is compared to those obtained using other well known methods, like Akaike criteria for time series or the singular values of the weighted projection matrix in the Stochastic Subspace Identification method. Finally, both simulated and measured vibration data are used to show the practicability of the derived technique. Finally, it is important to remark that the method can be used with any identification method working in the state space model.
\end{abstract}

\section{Introduction}

Vibration data are usually recorded in built structures to extract the modal parameters of these structures, among other applications. In practice, the modal parameters are often not the final goal for engineers, but only an intermediate result that can be used for a wide range of applications: model updating [1,2], structural health monitoring and damage detection $[3,4]$, load identification [5,6], and many others.

When performing vibration tests on large structures it is practically impossible to measure the excitation and the measured outputs are the only information that can be used by the system identification algorithms. In these cases it is assumed that the unknown input is a realization of a stochastic process, so stochastic system identification algorithms can be applied to the data. The terms output-only modal analysis and operational modal analysis are widely used to describe this technique where natural frequencies, damping ratios and mode shapes are estimated from output-only vibration data. 
But these identified modal parameters have to be validated, that is, a post-processing phase is needed to extract the real (physical) modes from them. Probably, the stabilization diagram is the most used post-processing tool for operational modal analysis. The stabilization diagram can be used for different purposes:

- To distinguish between stable physical modes and spurious modes: if we plot the modes for different model orders, the modes that have a similar behaviour at different orders are chosen to be physical modes of the structure [7].

- To select an order for the system model: by inspection, we choose the order of the system where all the stable modes are included, or at least, the modes we are interested in. The modal parameters are computed from the stable modes at the same order [8].

Fuzzy methods has been used to select the model order and related to this, to select valid system poles [9]. In [10], fuzzy clustering was introduced as a tool to automatically assess stabilization diagrams. Several algorithms were included, all based on the Fuzzy-C-Means clustering technique. Ref. [11] presented a method for the autonomous modal parameter identification and mode tracking by means of FRF measurements. The final model was selected using a fuzzy clustering approach, which separates physical from computational modes.

- Rather than trying to find one order and related state space model where all modes are stable, the modal parameters are computed at different orders selected from a stabilization diagram [12].

- To remove modes that represent non-white excitation or modes that represent, due to an under-estimation of the model order, a combination of several physical modes [13].

For these reasons, stabilization diagrams have become a standard tool in operational modal analysis. In this paper we propose some other techniques that can be used in the post-processing phase of the modal parameters. Rather than replacing the stabilization diagram, they are complementary tools and the joint use of them can give us valuable information.

For instance, the vibration contribution due to each identified mode can be estimated applying the Kalman filter. This is an important result if vibration levels have to be reduced by mitigation measures. Preliminary ideas were pointed out in [12]. We present here a detailed formulation of the procedure and how it can be used to compute the contribution of each mode to the measured vibration. This contribution is interpreted in terms of variance and energy.

A consequence of this is that we can compute the modal contribution at different model orders, for example, at the same orders used in the stabilization diagram. Then, the order of the state space model can be selected from these results.

The procedure can be used with any stochastic identification method that estimates a state space model from measured vibration data. The results presented here have been obtained with the stochastic subspace identification (SSI) method, one of the most robust and accurate system identification methods for operational modal analysis of structures. Two implementations with similar accuracy are available [12]: a covariance-driven (SSI-cov) and a data-driven (SSI-data) implementation. This method is based on the solution of the stochastic realization problem [14] and identifies state space models from (input and) output data by applying robust numerical techniques such as $Q R$ factorization, singular value decomposition (SVD) and least squares. A complete overview of data-driven subspace identification (both deterministic and stochastic) is provided in [15].

The organization of the text is as follows. In Section 2 it is indicated how vibrating structures can be represented by stochastic state space models, and how modal parameters are computed from these models. Section 3 presents a simulation structure that is used at different points of the work to check the proposed methods. In Section 4 the procedures to compute the contribution of the identified modes and to estimate the optimum order of the state space model are developed. Finally, the performance of the method is illustrated with operational data from a steel transmitter mast in Section 5 .

\section{Modal analysis in the state space model}

\subsection{State space equations}

The equation of motion for a $n_{d}$ degrees-of-freedom (DOF) linear, time invariant, viscously damped system subjected to external excitation is expressed as

$$
\mathcal{M} \ddot{q}(t)+\mathcal{D} \dot{q}(t)+\mathcal{K} q(t)=J u(t)
$$

where $\mathcal{M}, \mathcal{D}, \mathcal{K} \in \mathbb{R}^{n_{d} \times n_{d}}$ are the mass, damping and stiffness matrices, respectively; $J \in \mathbb{R}^{n_{d} \times n_{i}}$ is the excitation influence matrix that relates the $n_{i}$-dimensional input vector $u(t)$ to the $n_{d}$-dimensional response vector; $q(t)$ is the $n_{d}$-dimensional displacement response vector; dot denotes derivatives with respect to time.

By defining the state vector $x(t)=[q(t) \dot{q}(t)]^{T}$, Eq. (1) can be converted into the continuous state space form

$$
\dot{x}(t)=A_{c} x(t)+B_{c} u(t)
$$


where

$$
A_{c}=\left[\begin{array}{cc}
0 & I \\
-\mathcal{M}^{-1} \mathcal{K} & -\mathcal{M}^{-1} \mathcal{D}
\end{array}\right], \quad B_{c}=\left[\begin{array}{c}
0 \\
\mathcal{M}^{-1} J
\end{array}\right] .
$$

In practice, only a limited number of measurements are available in real structures; therefore, the dimension of the measurement output is less than or equal to the total number of degrees of freedom. Assuming we are measuring accelerations, the $n_{0}$-dimensional output vector $y(t)$ can be expressed as

$$
y(t)=C_{a} \ddot{q}(t)
$$

where $C_{a} \in \mathbb{R}^{n_{0} \times n_{d}}$ is the measurement location matrix corresponding to the acceleration responses of the structural system, composed of zeros and ones. We can rewrite the output vector into the continuous state space form

$$
y(t)=C_{c} x(t)+D_{c} u(t)
$$

where

$$
C_{c}=C_{a}\left[-\mathcal{M}^{-1} \mathcal{K} \quad-\mathcal{M}^{-1} \mathcal{D}\right] \quad D_{c}=C_{a} \mathcal{M}^{-1} J
$$

Eqs. (2) and (5) define the state space equation in continuous time:

$$
\begin{aligned}
& \dot{x}(t)=A_{C} x(t)+B_{c} u(t) \\
& y(t)=C_{c} x(t)+D_{c} u(t)
\end{aligned}
$$

where

$y(t) \in \mathbb{R}^{n_{0}}$ is the output vector;

$u(t) \in \mathbb{R}^{n_{i}}$ is the input vector;

$x(t) \in \mathbb{R}^{n_{s}}$ is the state vector;

$A_{c} \in \mathbb{R}^{n_{s} \times n_{s}}$ is the transition state matrix describing the dynamics of the system;

$B_{c} \in \mathbb{R}^{n_{s} \times n_{i}}$ is the input matrix;

$C_{c} \in \mathbb{R}^{n_{0} \times n_{s}}$ is the output matrix, which is describing how the internal state is transferred to the output vector $y(t)$;

$D_{c} \in \mathbb{R}^{n_{0} \times n_{i}}$ is the direct transmission matrix;

Eq. (7a) is known as the state equation and Eq. (7b) is known as the observation equation.

The continuous state space formulation is only useful for theoretical considerations because measurements are sampled at discrete time instants, so equations must be expressed in the discrete-time state space form. For the sampling of a continuous-time equation, we consider the input piecewise constant over the sampling period of length $\Delta t$ (ZeroOrder Hold assumption), that is

$$
\forall t \in\left[t_{k}, t_{k+1}\right)=[k \Delta t,(k+1) \Delta t) \Rightarrow x(t)=x\left(t_{k}\right)=x_{k}, u(t)=u\left(t_{k}\right)=u_{k}, y(t)=y\left(t_{k}\right)=y_{k} .
$$

Under this assumption, the continuous time state-space model (7a) and (7b) is converted to the discrete time statespace model:

$$
\begin{aligned}
& x_{k+1}=A x_{k}+B u_{k} \\
& y_{k}=C x_{k}+D u_{k} .
\end{aligned}
$$

The parameters are related to their continuous-time counterparts as (see for instance [16])

$$
A=e^{A_{c} \Delta t}, \quad B=(A-I) A_{c}^{-1} B_{c}, \quad C=C_{c}, \quad D=D_{c} .
$$

Up to now it has been considered that the system was only subjected to deterministic and known inputs, $u_{k}$. However, besides these measured inputs, there are always other that, although unknown, contribute to the system response. This unmeasurable influence is characterized as disturbance or noise. In system identification, system response disturbance might be caused by different phenomena. In any case, noise will always be present in measured data and should be therefore always take into account. It is necessary to extend the state space model $(9 \mathrm{a})$ and $(9 \mathrm{~b})$ including stochastic components, so a stochastic state space model is obtained:

$$
\begin{aligned}
& x_{k+1}=A x_{k}+B u_{k}+w_{k} \\
& y_{k}=C x_{k}+D u_{k}+v_{k}
\end{aligned}
$$

where $w_{k} \in \mathbb{R}^{n_{s}}$ is the process noise due to disturbances and modelling inaccuracies; $v_{k} \in \mathbb{R}^{n_{0}}$ is the measurement noise due to noise in sensor and acquisition system. Actually, they are both unmeasurable vector signals, but we assume that they 
are zero mean white noise sequences (discrete-time), with covariance matrices:

$$
E\left[\left[\begin{array}{l}
w_{p} \\
v_{p}
\end{array}\right]\left[w_{q}^{T} v_{q}^{T}\right]\right]=\left[\begin{array}{cc}
Q & S \\
S^{T} & R
\end{array}\right] \delta_{p q}
$$

where $E$ is the expected value operator and $\delta_{p q}$ is the Kronecker delta.

In the case of output-only testing, only the responses of a structure are measured, while the input sequence $u_{k}$ remains unmeasured. Thus, Eq. (11) results in a purely stochastic system:

$$
\begin{aligned}
& x_{k+1}=A x_{k}+w_{k} \\
& y_{k}=C x_{k}+v_{k} .
\end{aligned}
$$

Due to the stochastic nature of the state space Eqs. (13), it is only possible to predict the response of the system in terms of probability. For the state space model, this prediction is accomplished by the construction of the associated Kalman filter (see Appendix B). The Kalman filter is a computational scheme to estimate the states of a given state-space model in a statistically optimal manner.

Applying the Kalman filter, alternative equations for the stochastic state-space model (13) that are more suitable for some applications can be built: the so-called innovation form representation

$$
\begin{aligned}
& x_{k+1 \mid k}=A x_{k \mid k-1}+K \epsilon_{k} \\
& y_{k}=C x_{k \mid k-1}+\epsilon_{k}
\end{aligned}
$$

where $\epsilon_{k}$ are the innovations (B.3) and $K$ is the Kalman gain (B.4) under stationary conditions (see Appendix B); $x_{k \mid k-1}$ is the expected value of the state at time instant $k$ given the output measured to time instant $k-1$, that is, $\mathrm{E}\left[x_{k} \mid\left\{y_{1}, y_{2}, \ldots, y_{k-1}\right\}\right]$.

\subsection{System identification and modal analysis}

System identification can be described, in broad terms, as the construction of a dynamic model for a system based on experimental measurements. In the context of vibrating engineering structures, the system refers to structures such as buildings and bridges, and identification mostly involves the determination of modal parameters (the natural frequencies, damping ratios and mode shapes) [20].

System identification methods are classified into parametric model-based and non-parametric methods. Parametric model-based structural identification methods involve the use of mathematical models to represent structural system behaviour in either time or frequency domain. The benefits of using parametric models for structural identification include their direct relationship with physically meaningful quantities such as modal parameters, improved accuracy and resolution, and their suitability for analysis, vibrations prediction and control. Popular time-domain parametric models used for structural identification purposes include: state space models, ARX models, ARMAX models, etc. [21]. Many algorithms are available to estimate the parameters of such parametric models, e.g. prediction error minimization method (PEM), least squares estimation (LSE), instrumental variable method (IV), maximum likelihood estimation (MLE), subspace methods, and eigensystem realization algorithm (ERA). Non-parametric structural identification methods do not require a prior information on the nature of structural model, are very simple to apply but they require a huge amount of user interaction for finding the system modes. The most used non-parametric procedures using only ambient vibration responses are the peak picking (PP) and the complex mode indication function (CMIF), both in the frequency domain.

If we choose a state space model as the dynamic model for the structure, modal parameters can be computed from the estimated matrices $A$ and $C$ : the natural frequencies and modal damping ratios can be retrieved from the eigenvalues of $A$, and the mode shapes can be evaluated using the corresponding eigenvectors and the output matrix $C$. So the system identification problem when using the state space model given by Eq. (13) can be defined as the determination of the order $n_{\mathrm{S}}$ and the corresponding system matrices $A, C, Q, R$ and $S$ (up to within a similarity transformation) using the output measurements available for $N$ time steps, $\left\{y_{1}, y_{2}, \ldots, y_{N}\right\}$.

The eigenvalues of $A$ come in complex conjugate pairs and each pair represents one physical vibration mode. Assuming viscous damping, it is customary to express the eigenvalues of $A$ by (see [20, Section 3])

$$
\lambda_{j}=\exp \left[\left(-\zeta_{j} \omega_{j} \pm i \omega_{j} \sqrt{1-\zeta_{j}^{2}}\right) \Delta t\right],
$$

where $\omega_{j}$ is the natural frequency, $\zeta_{j}$ is damping ratio, and $\Delta t$ is the time step. Therefore

$$
\begin{aligned}
& \omega_{j}=\frac{\left|\ln \left(\lambda_{j}\right)\right|}{\Delta t}, \\
& \zeta_{j}=\frac{-\operatorname{Real}\left[\ln \left(\lambda_{j}\right)\right]}{\omega_{j} \Delta t} .
\end{aligned}
$$




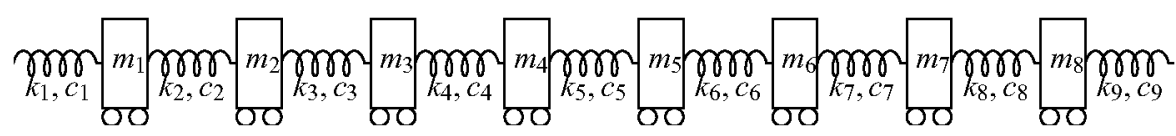

Fig. 1. Model of the simulated structure.

The $j$ th mode shape $\phi_{j} \in \mathbb{R}^{n_{o}}$ evaluated at sensor locations can be obtained using the following expression:

$$
\phi_{j}=C \psi_{j}
$$

where $\psi_{j}$ is the complex eigenvector of $A$ corresponding to the eigenvalue $\lambda_{j}$.

\section{Simulation example}

\subsection{Problem definition}

An eight DOF structure will be used along the paper for doing simulations and for validating the proposed method. The simulated structure consists of eight masses and nine springs and dashpots (Fig. 1), and the values chosen for matrices $\mathcal{M}$, $\mathcal{D}$ and $\mathcal{K}$ (see Eq. (1)) are the followings: $\mathcal{M}$ is equal to the identity matrix with eight rows and columns:

$$
\mathcal{K}=\left[\begin{array}{cccccccc}
2400 & -1600 & 0 & 0 & 0 & 0 & 0 & 0 \\
-1600 & 4000 & -2400 & 0 & 0 & 0 & 0 & 0 \\
0 & -2400 & 5600 & -3200 & 0 & 0 & 0 & 0 \\
0 & 0 & -3200 & 7200 & -4000 & 0 & 0 & 0 \\
0 & 0 & 0 & -4000 & 8800 & -4800 & 0 & 0 \\
0 & 0 & 0 & 0 & -4800 & 10400 & -5600 & 0 \\
0 & 0 & 0 & 0 & 0 & -5600 & 12000 & -6400 \\
0 & 0 & 0 & 0 & 0 & 0 & -6400 & 13600
\end{array}\right] \quad\left(\frac{\mathrm{N}}{\mathrm{m}}\right)
$$

$\mathcal{D}$ is built assuming Rayleigh damping by means of

$$
\mathcal{D}=0.680 \mathcal{M}+1.743 \times 10^{-4} \mathcal{K}\left(\frac{\mathrm{Ns}}{\mathrm{m}}\right) .
$$

The natural frequencies, mode shapes and damping ratios computed from matrices $\mathcal{M}, \mathcal{D}$ and $\mathcal{K}$, are presented in Fig. 2. The output signals have been generated using:

- Sampling frequency $f_{s}=50 \mathrm{~Hz}$ (sampling period $\Delta t=0.02 \mathrm{~s}$ ).

- Total duration of signals, $100 \mathrm{~s}$ ( $N=5000$ time steps).

- $u\left(t_{k}\right) \rightarrow N(0,1)$. This input has been applied to all the DOF by mean of matrix $J(J$ is a column vector composed of ones)

$$
\mathcal{M} \ddot{q}\left(t_{k}\right)+\mathcal{D} \dot{q}\left(t_{k}\right)+\mathcal{K} \ddot{q}\left(t_{k}\right)=J u\left(t_{k}\right), \quad k=1,2, \ldots, N .
$$

where $t_{k}=\{0, \Delta t, 2 \Delta t, \ldots, k \Delta t, \ldots,(N-1) \Delta t\}$.

Although the same input is applied to all DOF, all the modes are excited because the structure is non-symmetric (note that, if the structure were symmetric, the anti-symmetric modes would not be excited with this input).

- In real structures, not all DOF can be measured. In this case, we only use the accelerations of masses 1,4 and 8 . So matrix $C_{a}$ becomes

$$
C_{a}=\left[\begin{array}{llllllll}
1 & 0 & 0 & 0 & 0 & 0 & 0 & 0 \\
0 & 0 & 0 & 1 & 0 & 0 & 0 & 0 \\
0 & 0 & 0 & 0 & 0 & 0 & 0 & 1
\end{array}\right]
$$

- The observed values $y\left(t_{k}\right), k=1,2, \ldots, N$, are the sum of the structure response at the selected DOF, $C_{a} \ddot{q}\left(t_{k}\right)$, and a sensor Gaussian noise $a\left(t_{k}\right)$ with variance equal to the $20 \%$ of the largest acceleration response variance, $\sigma^{2}$ :

$$
\begin{aligned}
& y\left(t_{k}\right)=C_{a} \ddot{q}\left(t_{k}\right)+a\left(t_{k}\right), \quad a\left(t_{k}\right) \rightsquigarrow N\left(0, \Sigma^{2}\right), \\
& \Sigma^{2}=\sigma^{2} I_{n_{o} \times n_{o}}, \quad \sigma^{2}=0.20 \max \left(\frac{1}{N} \ddot{q}\left(t_{k}\right)^{T} \ddot{q}\left(t_{k}\right)\right) .
\end{aligned}
$$

\subsection{Operational modal analysis from the simulated accelerations}

We have applied the SSI-data method to identify the modal parameters of the simulated structure from the simulated acceleration, and the results are shown in Table 1 . The state space order used has been $n_{s}=16$, twice the number of modes. 

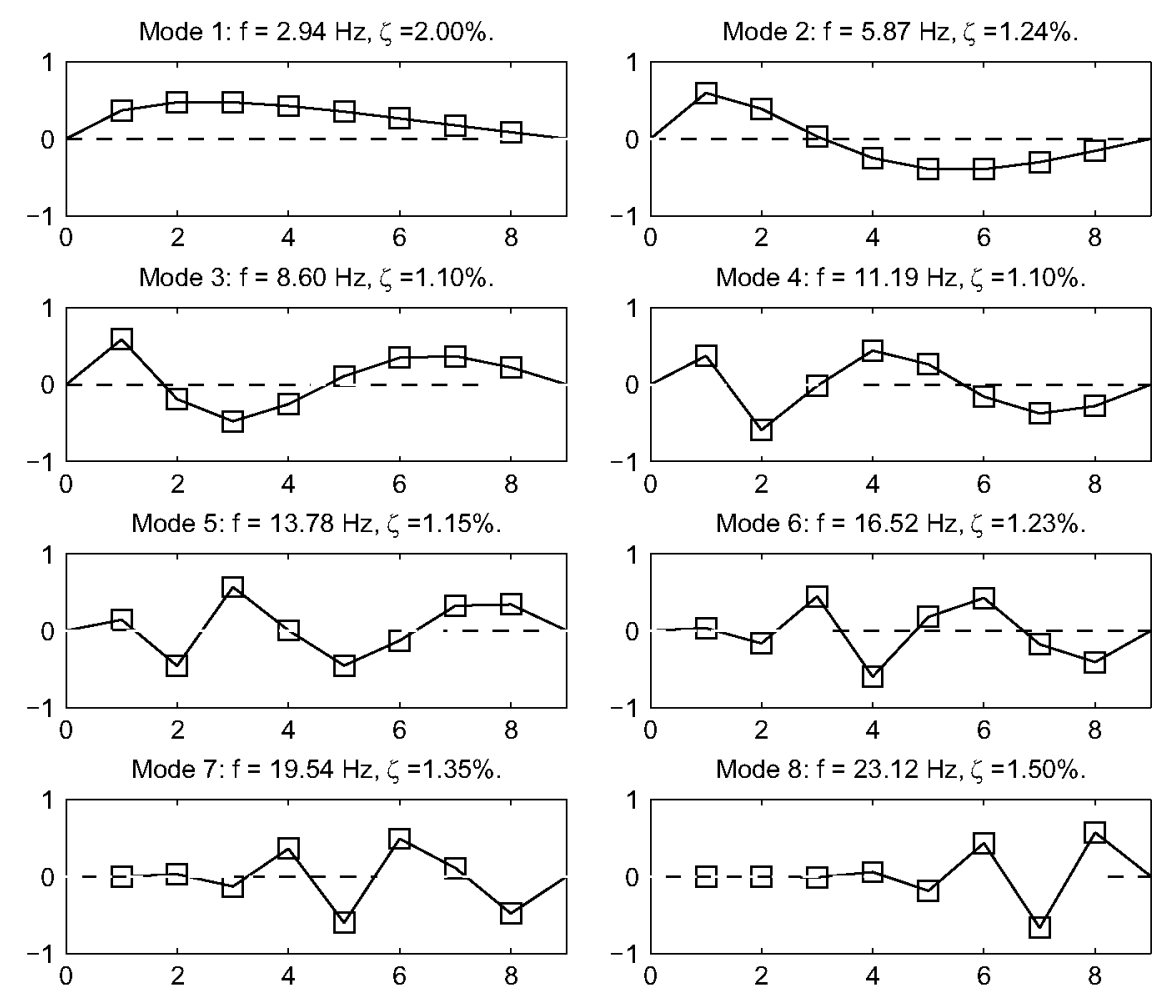

Fig. 2. Natural frequencies, damping ratios and mode shapes of the simulated structure.

Table 1

Theoretical (subscript th) and identified (SSI-data with order 16) natural frequencies and damping ratios.

\begin{tabular}{lcccc}
\hline Mode & $f_{\text {th }}(\mathrm{Hz})$ & $\hat{f}_{16}(\mathrm{~Hz})$ & $\zeta_{\text {th }}(\%)$ & $\hat{\zeta}_{16}(\%)$ \\
\hline 1 & 2.94 & 2.94 & 2.00 & 1.50 \\
2 & 5.87 & 5.86 & 1.24 & 1.39 \\
3 & 8.60 & 8.61 & 1.10 & 1.12 \\
4 & 11.19 & 11.19 & 1.10 & 0.95 \\
5 & 13.78 & 13.79 & 1.15 & 1.01 \\
7 & 16.52 & 16.52 & 1.23 & 1.30 \\
8 & 19.54 & 19.51 & 1.35 & 1.49 \\
\hline
\end{tabular}

In this example, the theoretical system order is known in advance, and good modal parameters have been obtained using this order. But in real structures we do not know the system order, because for continuous systems the number of modes inside the considered frequency interval can be very high. So when we choose an order for system identification we can underestimate or overestimate the correct order. In the first case, the identification method can compute wrong modes because it might try to fit the response due to close modes with only one. In the second case, we are forcing the identification method to compute more modes than the system has, so spurious modes are obtained. Furthermore, spurious modes can be obtained at any given order because of modelling errors.

The most popular tool for differentiating system modes from spurious modes is the stabilization diagram [22]. A stabilization diagram is simply a plot of various model orders versus the frequencies identified at each of these orders. The motivation is that a system mode should show up with consistent frequency, damping and mode shape at various model orders whereas the spurious ones could be expected to show a somewhat more erratic behaviour.

The actual implementation of this strategy can be executed by initially choosing a sufficiently high order for the state space model, and then gradually reduce the order of the model. System identification is performed with every model order so this procedure yields a set of modal parameters for each selected order. Parameters that belong to two different model orders are then compared according to some preset criteria such as

$$
\frac{\left|f_{p i}-f_{q j}\right|}{f_{p i}} \leq \varepsilon_{f},
$$




$$
\begin{aligned}
& \frac{\left|\zeta_{p i}-\zeta_{q j}\right|}{\zeta_{p i}} \leq \varepsilon_{\zeta}, \\
& 1-M A C\left(\phi_{p i}, \phi_{q j}\right) \leq \varepsilon_{M A C},
\end{aligned}
$$

where $\varepsilon_{f}, \varepsilon_{\zeta}, \varepsilon_{\text {MAC }}$ are tolerance limits to decide if mode $i$ estimated from model order $p$ is the same that mode $j$ estimated from model order $q$ (it is usual to take $q=p+1$, that is, to compare consecutive model orders). MAC (modal assurance criterion) shows the degree of correlation between two vectors and it is computed as

$$
\operatorname{MAC}\left(\phi_{1}, \phi_{2}\right)=\frac{\left|\phi_{1}^{H} \phi_{2}\right|^{2}}{\left(\phi_{1}^{H} \phi_{1}\right)\left(\phi_{2}^{H} \phi_{2}\right)}
$$

where $(\bullet)^{H}$ means Hermitian operator.

This procedure is repeated for all available sets of modal parameters identified at each order in a sequential manner, and finally, the frequencies are plotted against their corresponding model orders, distinguishing between stable and unstable modes. It is usual to add to the graphic the power spectral density plot of a selected channel, or the Averaged Normalized Power Spectral Density (ANPSD)

$$
\operatorname{ANPSD}(f)=\frac{1}{n_{o}} \sum_{i=1}^{n_{o}} \frac{P S D(f)_{i}}{\sum_{j=1}^{N} \operatorname{PSD}\left(f_{j}\right)_{i}}
$$

where $P S D(f)_{i}$ is the power spectral density of channel $i$. In this case, auto-spectra functions are normalized and averaged to obtain an average spectra density function from all channels that, normally, shows all resonance frequencies of the system. The stable frequencies should be reflected by the peaks of the ANPSD plot.

Fig. 3 shows the stabilization diagram of the simulated structure, using orders from 2 to 30 by steps of two. The modal parameters for each order have been identified using SSI-data. The stable modes, those verifying $\varepsilon_{f}=0.02, \varepsilon_{\zeta}=0.05$, $\varepsilon_{\text {MAC }}=0.05$ simultaneously, have been plotted with $\mathbf{a} \oplus$, while the unstable modes have been plotted with a cross + . The figure also include the plot of the ANPSD.

Probably, the stabilization diagram is the most used post-processing tool for operational modal analysis. In the next section we propose other tools based on the application of the Kalman filter.

\section{Mode contribution and selection of the order of the state space model}

\subsection{Computing the vibration due to the modes}

It is well known that the state vector of a system is not unique because we can transform the state vector $x_{k}$ into another one $z_{k}$ by a linear transformation as follows:

$$
x_{k}=T z_{k} \text {, }
$$

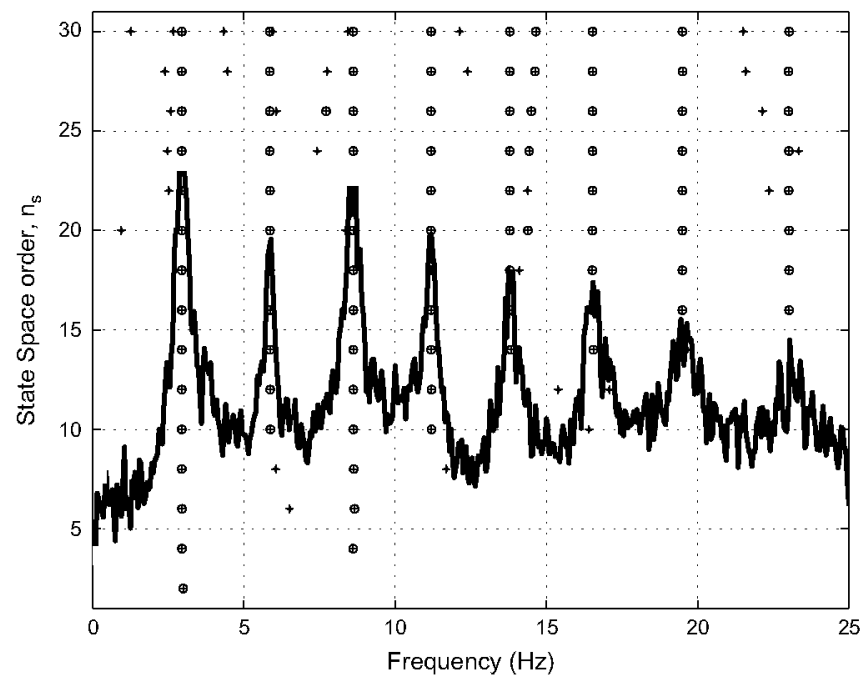

Fig. 3. Stabilization diagram corresponding to the simulated accelerations. The criteria are $2 \%$ for frequencies, $5 \%$ for damping ratios, $5 \%$ for mode shape vectors $(\mathrm{MAC}) . \oplus$, stable mode; +, unstable mode. The ANPSD is plotted in light gray. 
where $T$ is the transformation matrix. Replacing this condition into (13) and pre-multiplying by $T^{-1}$

$$
\begin{aligned}
& z_{k+1}=A_{1} z_{k}+T^{-1} w_{k} \\
& y_{k}=C_{1} z_{k}+v_{k},
\end{aligned}
$$

with

$$
A_{1}=T^{-1} A T, \quad C_{1}=C T .
$$

This state representation yields the same input-output behaviour that of (13). An important state vector is defined by the transformation $x_{k}=V \mu_{k}$, where $V$ is the matrix of eigenvectors of the eigenvalue problem

$$
A v=\lambda v, \quad \lambda \in \mathbb{R}, \quad v \in \mathbb{R}^{n_{s} \times 1} .
$$

The solution of this problem is a set of $n_{5}$ eigenvalues $\lambda_{j}$ and eigenvectors $v_{j}$. It is usual to group the eigenvalues in a diagonal matrix $A_{0}$ and the eigenvectors (by columns) in the matrix $V$ verifying

$$
A V=V A_{0} \quad \Rightarrow \quad A_{0}=V^{-1} A V
$$

The state space representation obtained

$$
\begin{aligned}
& \mu_{k+1}=A_{0} \mu_{k}+V^{-1} w_{k} \\
& y_{k}=C_{0} \mu_{k}+v_{k}
\end{aligned}
$$

is called the state space equations in modal form, because $\mu_{k}$ are the modal states. The same procedure can be followed with the innovation form representation, Eq. (14), obtaining

$$
\begin{aligned}
& \mu_{k+1 \mid k}=A_{0} \mu_{k \mid k-1}+K_{0} \epsilon_{k} \\
& y_{k}=C_{0} \mu_{k \mid k-1}+\epsilon_{k}
\end{aligned}
$$

where

$$
x_{k \mid k-1}=V \mu_{k \mid k-1}, \quad A_{0}=V^{-1} A V, \quad C_{0}=C V, \quad K_{0}=V^{-1} K .
$$

An important advantage of using Eqs. (27) instead of (26) is that we can compute the expected states $\mu_{k \mid k-1}$ using the Kalman filter (Appendix B) if we know the matrices $\left\{A_{0}, C_{0}, Q_{0}, R_{0}, S_{0}\right\}$. On the contrary, we cannot compute the states $\mu_{k}$ because we do not know the error sequences, $w_{k}$ and $v_{k}$.

Substituting (27b) into (27a), Eq. (27) can be also expressed as

$$
\begin{aligned}
& \mu_{k+1 \mid k}=\left(A_{0}-K_{0} C_{0}\right) \mu_{k \mid k-1}+K_{0} y_{k} \\
& y_{k}=C_{0} \mu_{k \mid k-1}+\epsilon_{k}
\end{aligned}
$$

where the innovations $\epsilon_{k}$ are the prediction errors, and $C_{0} \mu_{k \mid k-1}$ is the one-step-ahead predicted output.

On the other hand, taking into account Eq. (18), the matrix $C_{0}=C V$ is an estimate of the mode shapes, so $C_{0} \mu_{k \mid k-1}$ is an estimate of the response due to the modes:

$$
\begin{aligned}
& y_{k}=C_{0} \mu_{k \mid k-1}+\epsilon_{k} \\
& \Rightarrow y_{k}=y_{k}^{m}+y_{k}^{\epsilon}
\end{aligned}
$$

where

- $y_{k}^{m}=C_{0} \mu_{k \mid k-1}$ is the acceleration due to the modes given by the pair $\left\{A_{0}, C_{0}\right\}$, or what is the same, the modes given by $\{A, C\}$.

- $y_{k}^{\epsilon}=\epsilon_{k}$ is the error term of the acceleration, that is, the acceleration needed by $y_{k}^{m}$ to be equal to $y_{k}$.

Since $A_{0}$ is a diagonal matrix, we can separate the acceleration of each individual mode: the diagonal elements of matrix $A_{0}$ come in complex conjugate pairs and each pair represents one physical vibration mode (see Eq. (15)), what can be used to split Eq. (28) as

$$
\begin{aligned}
y_{k} & =C_{0} \mu_{k \mid k-1}+\epsilon_{k}=C_{0} I_{m_{1}} \mu_{k \mid k-1}+C_{0} I_{m_{2}} \mu_{k \mid k-1}+\cdots+C_{0} I_{m_{n_{m}}} \mu_{k \mid k-1}+\epsilon_{k} \\
& \Rightarrow y_{k}=y_{k}^{m}+y_{k}^{\epsilon}=y_{k}^{m_{1}}+y_{k}^{m_{2}}+\cdots+y_{k}^{m_{n_{m}}}+y_{k}^{\epsilon}
\end{aligned}
$$

where $I_{m_{n}}$ is a matrix composed of zeros, except the diagonal elements corresponding to eigenvalue $n$ and its complex conjugate, which are equal to 1 (so the sum of matrices $I_{m_{n}}$ is equal to the identity matrix); $y_{k}^{m_{n}}$ represents the output values (acceleration in this example) corresponding to the identified mode $n$ at time instant $k$; $n_{m}$ is the number of identified modes. In principle, these modes should correspond to $n_{m}$ complex conjugate pairs of eigenvalues $\left(n_{m}=n_{s} / 2\right)$. However, some real values can be present in the eigenvalues of $A\left(n_{m} \neq n_{s} / 2\right)$, and the matrix $I_{m}$ associated to these eigenvalues will have only a diagonal element equal to one. 
In summary, given the measured accelerations $\left\{y_{1}, y_{2}, \ldots, y_{N}\right\}$, and the state space model matrices estimated from them, $\{\hat{A}, \hat{C}, \hat{Q}, \hat{R}, \hat{S}\}$, we can compute the acceleration of the modes present in $\{\hat{A}, \hat{C}\}$ applying the Kalman filter.

In Fig. 4 we have plotted the acceleration of mass number 1 for the first three modes of the simulated structure (theoretical modal acceleration). We have also plotted the acceleration of this mass, but computed using the matrices identified with SSI method $\left(n_{s}=16\right)$ and the proposed procedure (estimated modal acceleration).

The theoretical modal acceleration is the acceleration corresponding to each theoretical mode when applying the mode-superposition method to solve the equation of motion for an $n_{d}$-DOF system, Eq. (1). The method consists in defining a coordinate transformation

$$
q(t)=\Phi \eta(t)=\left[\begin{array}{llll}
\phi_{1} & \phi_{2} & \cdots & \phi_{n_{d}}
\end{array}\right]\left[\begin{array}{c}
\eta_{1}(t) \\
\eta_{2}(t) \\
\cdots \\
\eta_{n_{d}}(t)
\end{array}\right],
$$

where $\eta(t)$ are the normal (modal) coordinates, and $\Phi$ is the mode-shape matrix (formed by the mode shapes $\phi_{n}$ by columns). The $n_{d}$ coupled differential equations defined by (1) are then transformed into a set of $n_{d}$ independent second order differential equations:

$$
\ddot{\eta}_{n}(t)+2 \zeta_{n} \omega_{n} n \dot{\eta}_{n}(t)+\omega_{n}^{2} \eta_{i}(t)=\varphi_{n}(t), \quad n=1,2, \ldots, n_{d},
$$

where $\omega_{n}, \zeta_{n}$ are the natural frequency and damping ratio of mode $n$, respectively; $\varphi_{n}(t)$ is the row $n$ of the modal forces, $\left(\Phi^{T} M \Phi\right)^{-1}\left(\Phi^{T} J u(t)\right)$. These $n_{d}$ differential equations can be solved obtaining $n_{d}$ displacements in modal coordinates, $\eta_{n}(t)$; the solution in the original coordinates, $q(t)$, is then computed by mean of Eq. (31) and the $n_{d}$ individual solutions $\eta_{n}(t)$ :

$$
q(t)=\Phi \eta(t)=\phi_{1} \eta_{1}(t)+\phi_{2} \eta_{2}(t)+\cdots+\phi_{n_{d}} \eta_{n_{d}}(t), \quad q(t) \in \mathbb{R}^{n_{d}}
$$

The acceleration is obtained similarly

$$
\begin{aligned}
\ddot{q}(t)= & \Phi \ddot{\eta}(t)=\phi_{1} \ddot{\eta}_{1}(t)+\phi_{2} \ddot{\eta}_{2}(t)+\cdots+\phi_{n_{d}} \ddot{\eta}_{n_{d}}(t) \\
& \Rightarrow \ddot{q}(t)=\ddot{q}^{m_{1}}(t)+\ddot{q}^{m_{2}}(t)+\cdots+\ddot{q}^{m_{n_{d}}}(t)
\end{aligned}
$$

where $\ddot{q}^{m_{n}}(t)$ is the theoretical acceleration corresponding to mode $n, \ddot{q}^{m_{n}}(t)=\phi_{n} \ddot{\eta}_{n}(t)$. These values have been obtained at discrete time instants, $t_{k}=k \Delta t$

$$
\ddot{q}\left(t_{k}\right)=\ddot{q}^{m_{1}}\left(t_{k}\right)+\ddot{q}^{m_{2}}\left(t_{k}\right)+\cdots+\ddot{q}^{m_{n_{d}}}\left(t_{k}\right), \quad k=0,1,2, \ldots, N-1 .
$$
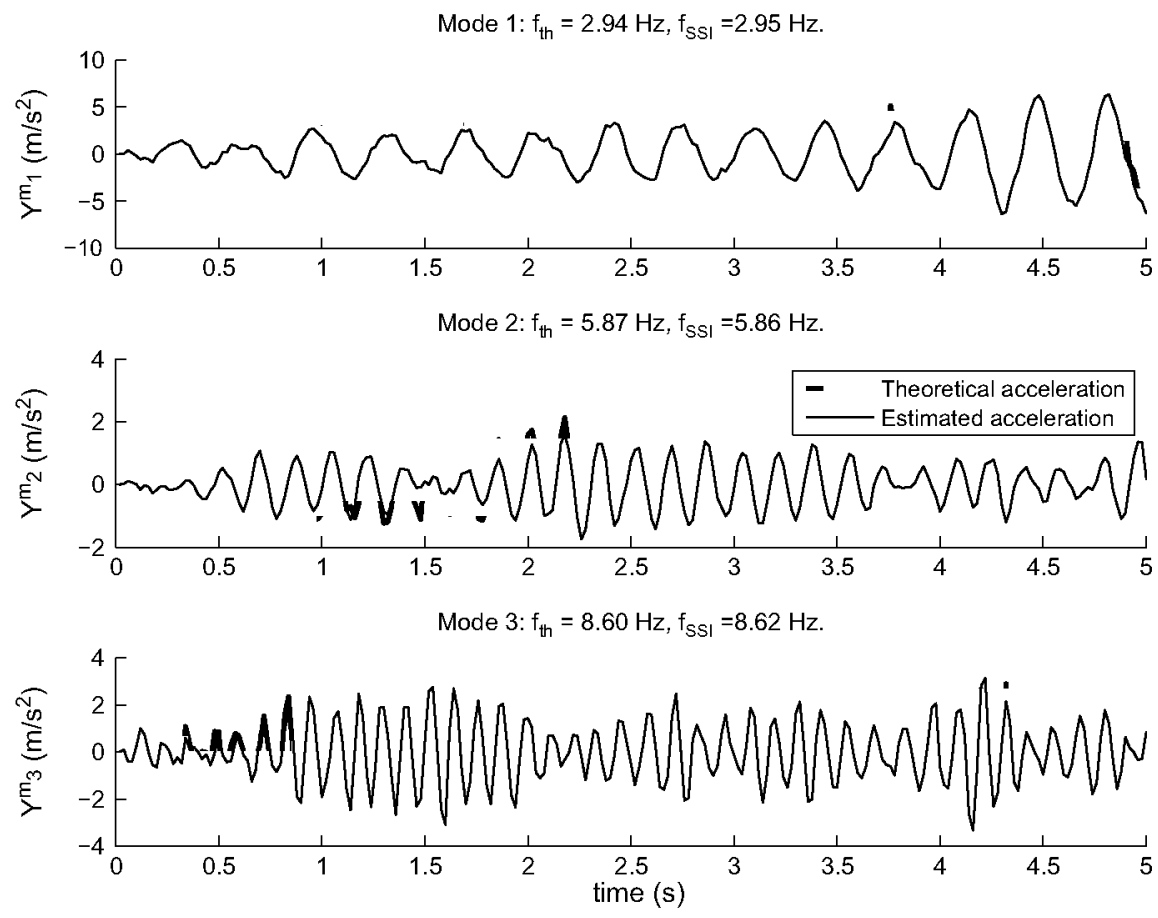

Fig. 4. Theoretical acceleration and estimated acceleration for the three first modes of the simulated structure (mass number 1). 
The acceleration computed using Eq. (30) constitutes an estimation of the corresponding theoretical modal acceleration, that is

$$
\hat{\bar{q}}^{m_{n}}\left(t_{k}\right)=y_{k}^{m_{n}}=C_{0} I_{m_{n}} \mu_{k \mid k-1}, \quad k=0,1,2, \ldots, N-1 .
$$

Fig. 4 shows that both accelerations, the theoretical and the estimated one, agree quite well for the simulated structure.

\subsection{Contribution of each identified mode to the measured acceleration}

Given the measured accelerations, we can use the SSI method to fit the state space equations (13a) and (13b) to them. Modal parameters can be computed from this model (and more specifically from the matrices $A$ and $C$ ), and in the precedent section we have presented a procedure to compute the acceleration due to each identified mode. The idea now is to quantify in some way the importance of this modal acceleration, that is, to quantify the contribution of each modal acceleration to the total measured acceleration.

We propose the following procedure: using Eq. (28) we can estimate the modal acceleration and the error acceleration as well for each time step. We need the starting value $\mu_{1 \mid 0}$ to perform the iterations; if the algorithm used to estimate matrices $A, C, Q, R$ and $S$ does not estimate this parameter, we can assume $\mu_{1 \mid 0}=0$ (that is what we have done in this work). Rearranging these values in matrix form

$$
\begin{aligned}
& {\left[\begin{array}{llll}
y_{1} & y_{2} & \cdots & y_{N}
\end{array}\right]=\left[\begin{array}{llll}
y_{1}^{m} & y_{2}^{m} & \cdots & y_{N}^{m}
\end{array}\right]+\left[\begin{array}{llll}
y_{1}^{\epsilon} & y_{2}^{\epsilon} & \cdots & y_{N}^{\epsilon}
\end{array}\right] \Rightarrow} \\
& Y=Y^{m}+Y^{\epsilon} .
\end{aligned}
$$

Multiplying by the transpose of $Y$

$$
Y Y^{T}=Y^{m} Y^{T}+Y^{\epsilon} Y^{T}
$$

We are interested in the diagonal elements of $Y^{m} Y^{T}$ and $Y^{\epsilon} Y^{T}$ : for example, the element $(n, n)$ of matrix $Y^{m} Y^{T}$ is the covariance between the acceleration measured at channel $n$ and the acceleration at channel $n$ due to the modes. Retaining only the diagonal elements

$$
\left(Y Y^{T}\right)_{D}=\left(Y^{m} Y^{T}\right)_{D}+\left(Y^{\epsilon} Y^{T}\right)_{D}
$$

where $(\bullet)_{D}$ is the diagonal operator: $(M)_{D}$ is a matrix consisting of the diagonal elements of the matrix $\mathrm{M}$ and zeros elsewhere. Normalizing the above equation:

$$
\begin{aligned}
& \left(Y Y^{T}\right)_{D}^{-1}\left(Y Y^{T}\right)_{D}=\left(Y Y^{T}\right)_{D}^{-1}\left(Y^{m} Y^{T}\right)_{D}+\left(Y Y^{T}\right)_{D}^{-1}\left(Y^{\epsilon} Y^{T}\right)_{D} \Rightarrow \\
& \{1\}_{n_{o}}=\Delta_{m}+\Delta_{\epsilon},
\end{aligned}
$$

where

- $\{1\}_{n_{0}}$ is a column vector with $n_{0}$ elements, all equal to one;

- $\Delta_{m}$ is a column vector formed by the diagonal of matrix $\left(Y Y^{T}\right)_{D}^{-1}\left(Y^{m} Y^{T}\right)_{D}$.

- $\Delta_{\epsilon}$ is a column vector formed by the diagonal of matrix $\left(Y Y^{T}\right)_{D}^{-1}\left(Y^{\epsilon} Y^{T}\right)_{D}$

According to Eq. (34), we define the element $n$ of vector $A_{m}$ as the contribution of the identified modes to the channel $n$ of the output vector, and $\Delta_{\epsilon}(n)$ as the contribution of the error to the channel $n$. The contribution of the modes plus the contribution of the error (always present due to the approximative nature of the Kalman filter) is equal to one in each channel.

We also define the global contribution of the modes, $\delta_{m}$, and the global contribution of the error, $\delta_{\epsilon}$, as the mean value of the corresponding vector

$$
\delta_{m}=\frac{1}{n_{o}} \sum_{n=1}^{n_{o}} \Delta_{m}(n), \quad \delta_{\epsilon}=\frac{1}{n_{o}} \sum_{n=1}^{n_{o}} \Delta_{\epsilon}(n) \Rightarrow \delta_{m}+\delta_{\epsilon}=1 .
$$

If we use Eq. (30) in place of (28), we obtain the contribution of each mode individually

$$
Y=Y^{m_{1}}+Y^{m_{2}}+\cdots+Y^{m_{n_{m}}}+Y^{\epsilon},
$$

and following the same procedure

$$
\{1\}_{n_{o}}=\left(Y Y^{T}\right)_{D}^{-1}\left(Y^{m_{1}} Y^{T}\right)_{D}+\left(Y Y^{T}\right)_{D}^{-1}\left(Y^{m_{2}} Y^{T}\right)_{D}+\cdots+\left(Y Y^{T}\right)_{D}^{-1}\left(Y^{m_{n_{m}}} Y^{T}\right)_{D}+\left(Y Y^{T}\right)_{D}^{-1}\left(Y^{\epsilon} Y^{T}\right)_{D} .
$$

Retaining only the diagonal of the matrices

$$
\begin{aligned}
& \{1\}_{n_{o}}=\Delta_{m_{1}}+\Delta_{m_{2}}+\cdots+\Delta_{m_{n_{m}}}+\Delta_{\epsilon}, \\
& \delta_{m_{k}}=\frac{1}{n_{o}} \sum_{n=1}^{n_{o}} \Delta_{m_{k}}(n) \Rightarrow \sum_{k=1}^{n_{m}} \delta_{m_{k}}+\delta_{\epsilon}=\delta_{m}+\delta_{\epsilon}=1 .
\end{aligned}
$$


$\mathrm{n}_{\mathrm{s}}=8, \quad \delta_{\mathrm{m}}=0.61354$
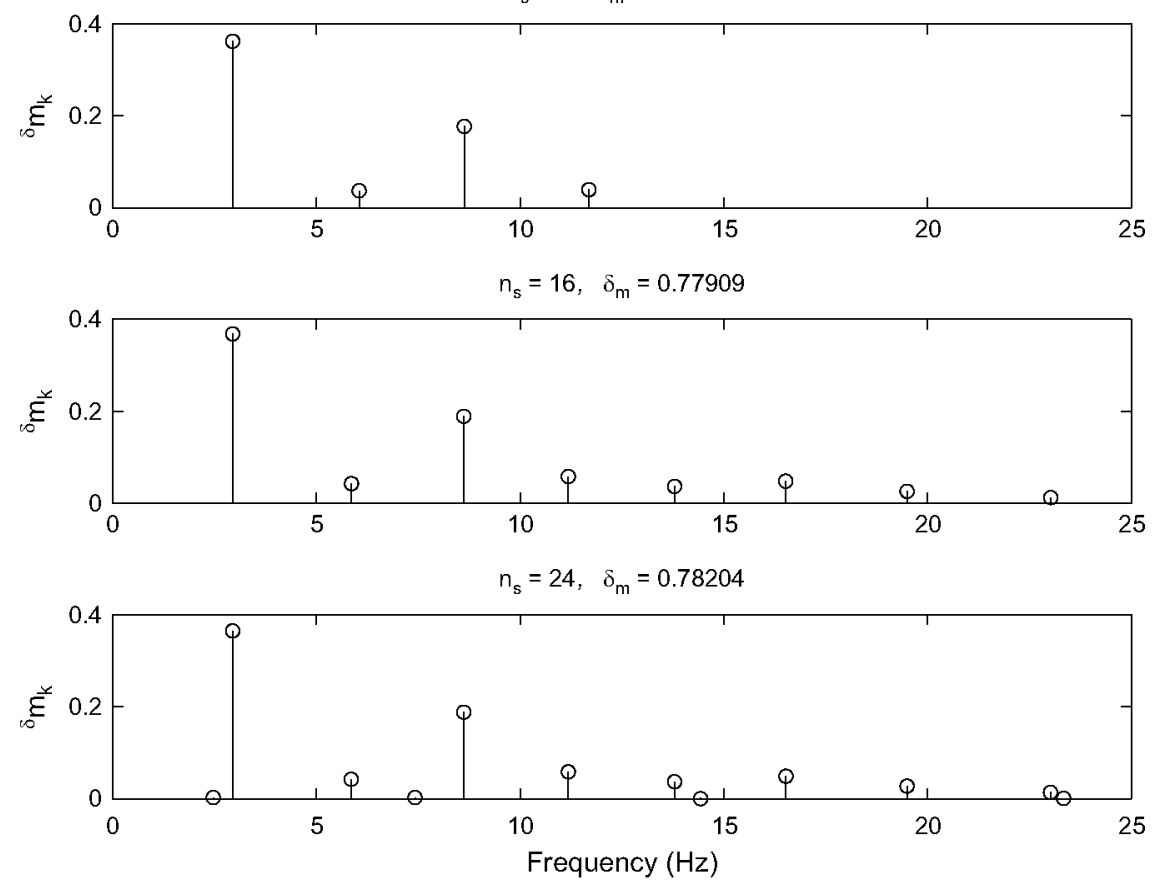

Fig. 5. Modal contribution of the identified modes for different model orders.

These last equations show the contribution of each identified mode to the measured signal: $A_{m_{k}}(n)$ is the contribution of the identified mode $k$ to the channel $n$ of the output vector, and $\delta_{m_{k}}$ is the total contribution of mode $k$ to the output vector. For example, in Fig. 5 we have plotted the contribution of the identified modes at the exact model order ( $n_{s}=16$ ) and at orders lower and higher than the exact. The principal conclusions derived from this figure are as follows:

- The modal contribution, $\delta_{m}$, increases when we include more modes, but for model orders greater than the theoretical one $\left(n_{s}=16\right)$, the modal contribution is almost constant $\left(n_{s}=8 \rightarrow \delta_{m}=0.6135 ; n_{s}=16 \rightarrow \delta_{m}=0.7791 ; n_{s}=24 \rightarrow \delta_{m}=0.7820\right)$.

- The simulated signal was created adding $20 \%$ of noise to the system acceleration, and the modal contribution obtained (the contribution of the modes to the simulated signal) is near to $80 \%$.

- The modes with greater contribution are those corresponding to the frequencies $f_{1}=2.94 \mathrm{~Hz}$ and $f_{3}=8.60 \mathrm{~Hz}$.

- It is important to note that the contribution for one mode remains constant for different model orders. For example, the mode corresponding to $2.94 \mathrm{~Hz}: \delta_{m_{1}}=0.3615$ at $n_{5}=8, \delta_{m_{1}}=0.3675$ at $n_{s}=16$ and $\delta_{m_{1}}=0.3649$ at $n_{s}=24$.

- At model order $n_{s}=24$ there are four modes that are spurious (see stabilization diagram, Fig. 3 ). The modal contribution of these spurious modes is almost zero.

- Mode number $8, f_{8}=23.12 \mathrm{~Hz}$, is the mode with lower modal contribution, $\delta_{m_{8}}=0.0368$. In fact, mode 8 is a weakly excited mode, the mode with the lower power at the spectrum included in the stabilization diagram. The modal contribution measures what is the contribution of the identified modes to the measured signal. The contribution of weakly excited modes is obviously low. On the other hand, if the mode is spurious, the contribution to the signal is low as well. So it is difficult to difference between spurious and weakly excited modes attending to the modal contribution.

Contribution can be also thought in terms of variance. Let be, for example, a signal $\gamma_{k}$ obtained as the sum of two other signals $\gamma_{k}=\alpha_{k}+\beta_{k}$. Multiplying by the transpose of $\gamma_{k}$ and dividing by the total number of data, $N$

$$
\frac{1}{N} \sum_{k=1}^{N} \gamma_{k} \cdot \gamma_{k}^{T}=\frac{1}{N} \sum_{k=1}^{N} \alpha_{k} \cdot \gamma_{k}^{T}+\frac{1}{N} \sum_{k=1}^{N} \beta_{k} \cdot \gamma_{k}^{T} \Rightarrow \operatorname{var}\left(\gamma_{k}\right)=\operatorname{cov}\left(\gamma_{k}, \alpha_{k}\right)+\operatorname{cov}\left(\gamma_{k}, \beta_{k}\right) .
$$

So Eq. (33) can be seen as the contribution of the modes and the error to the variance of the measured accelerations, $y_{k}$. Fig. 6 presents this graphically, where five seconds of the accelerations due to the identified modes $\left(n_{s}=16\right)$ at channel number one (mass number 1) are shown. At this model order the total modal contribution is given only by four modes, which are shown in the figure from increasing value of modal contribution and from top to bottom. The left column presents the total measured acceleration (in gray) and the acceleration due to each estimated mode (in black); the right column presents again the total measured acceleration and the sum of the acceleration of this mode and the precedent modes. We see in this figure that the mode with higher modal contribution at channel 1 (that is, $\Delta_{m_{k}}(1)$ ) contributes more to the variability (or variance) of the total signal. 

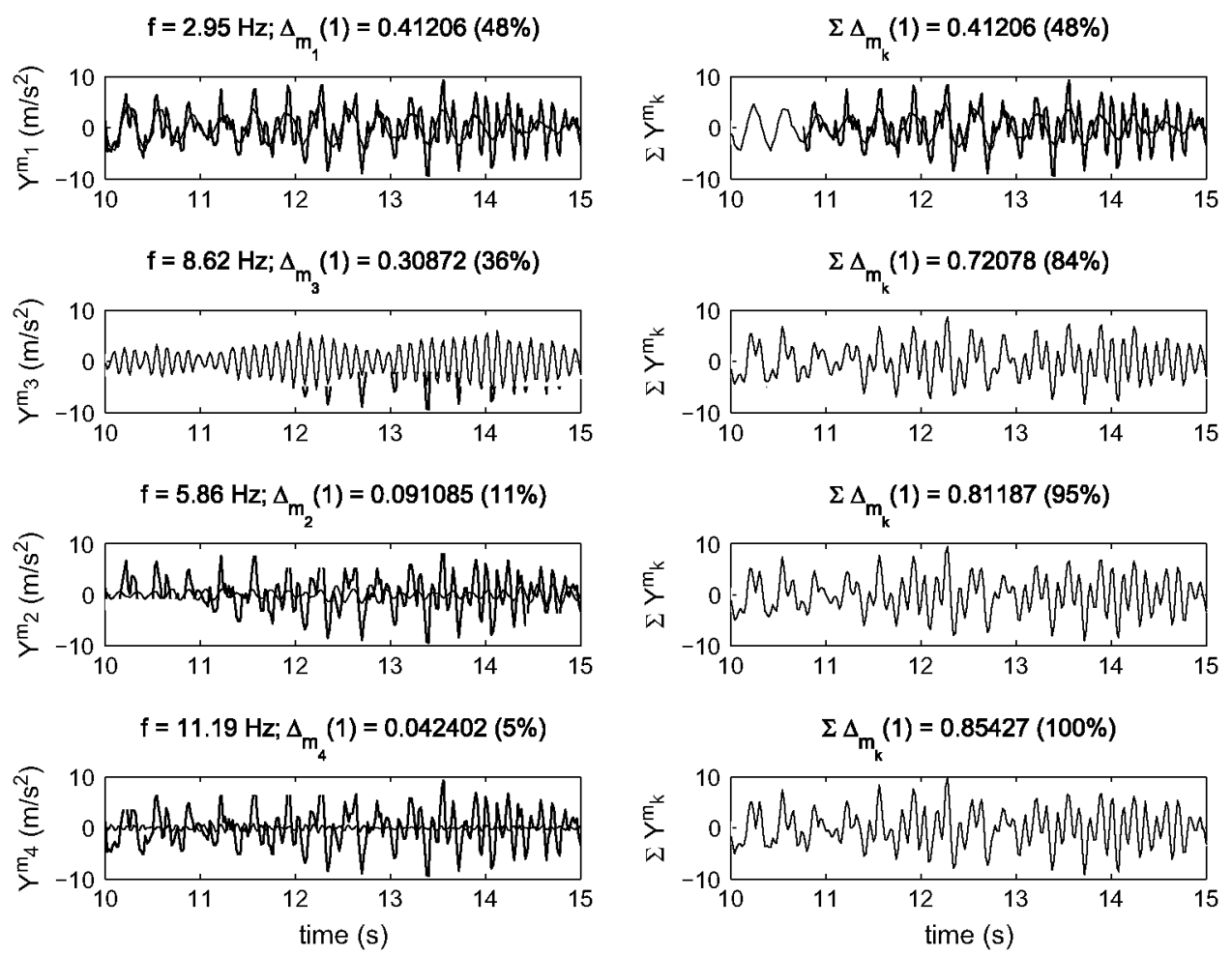

Fig. 6. Total measured acceleration (gray) and estimated acceleration (black) for the three first modes of the simulated structure (mass number 1).

But the variance of a signal can be computed by mean of the well known formula $\operatorname{Var}(X)=E\left(X^{2}\right)-(E(X))^{2}$, where $E\left(X^{2}\right)$ is a measure of the energy of the signal. In structure vibration it is usual to take zero-mean signals, so $\operatorname{Var}(X)=E\left(X^{2}\right)$ and the modal contribution might be also considered as a measure of the energy added by each mode to the total energy of the signal.

The process of isolating the vibration corresponding to each mode and computing its contribution to the measured signal can be used, for instance, as an alternative to the modal transfer norm [13] to remove mathematical modes and noise modes from the model. The mode contribution, like the modal transfer norm, is a measure for the error that is made when a mode is removed from the model. The mode contribution might also be used in many applications: in the design of devices for reducing the dynamic response of the structure, like Tuned Mass Dampers, because we identify the modes that are mainly responsible for the observed vibrations; the importance of these modes, both overall and at every sensor point is quantified. Another use can be the reduction of the model order, keeping only the modes with higher contributions.

\subsection{Contribution of the error to the measured acceleration and order selection}

For low model orders only a few modes of the system are identified and thus the modal contribution is low. However, for very high model orders, all the principal modes (those that sum almost the total modal contribution) are identified. Therefore, the modal contribution becomes constant for high order models, because the new identified modes do not add significant contribution.

The contrary happens whether we think in terms of error (the error are the innovations from Eq. (14)), because the contribution of the error and the modal contribution are directly related by means of Eq. (34). With low model orders, the contribution of the error is high, which means that dynamics are still present in the residuals. Nevertheless, for high order models these dynamics have been included in matrices $A$ and $C$ and the error contribution does not decrease although we increase the order.

This observation suggests us that the point where the error contribution (and the modal contribution as well) becomes constant is indicative of the model order because at this point all the principal modes have been taken into account, and although we increase the model order, we do not reduce the error of the equations. So if we plot the error contribution versus the system order we obtain a high value for the lower model orders and, as we increase the model order, the contribution of the error decreases until a point where it becomes constant: this point is proposed as the model order for the system. Of course, we should say a region instead of a point, because this transition to a constant value might be smooth and not sharp, depending on the characteristics of the system and the input. 
Fig. 7(a) shows the error and the modal contribution for the simulated structure and for orders comprised between 2 and 30. We can distinguish two clear tendencies: from order 4 to order 16, the error contribution decreases; from order 16 to order 30 the error contribution is constant. In this simulated example, the point where the error contribution became constant is $n_{s}=16$, so this is the proposed system order. In this case, the proposed order match the correct order of the system ( 8 modes).

The choice of the order for a system when using real data is far from a trivial task. Many procedures have been proposed in the literature, and between them we highlight:

- The Akaike criteria (AIC) and Schwarz criteria (SIC) in time series analysis [23-25]. The AIC and SIC have their separate theoretical justifications and properties. The AIC is derived by considering the principles of maximum likelihood and of negative entropy. The maximum likelihood approach itself cannot be used to choose a model dimension since the likelihood is maximised at the highest dimension under consideration. However, the concept of negative entropy extends the maximum likelihood approach and is appropriate for model selection. The model having minimum AIC should have minimum prediction set error, at least asymptotically. On the other hand, the SIC is minimised at the model order having the highest posterior probability, also asymptotically.

In practice, both criteria differ in terms of the penalty attached to increasing the model order, but SIC usually gives a lower model order than AIC. For state space models:

$$
\begin{aligned}
& A I C=-2 \log L_{Y_{N}}+2 P \\
& S I C=-2 \log L_{Y_{N}}+2 P \log N
\end{aligned}
$$

where $P$ is the number of free parameters in the model and $L_{Y_{N}}$ is the likelihood given $N$ measurements of the outputs $Y_{N}=\left\{y_{1}, y_{2}, \ldots, y_{N}\right\}$. The likelihood can be computed using the innovations $\left\{\epsilon_{1}, \epsilon_{2}, \ldots, \epsilon_{N}\right\}$ (see for example [17,18]), which are defined by Eq. (B.3). The innovations are independent Gaussian random vectors, $\epsilon_{t} \rightarrow N\left(0, \Sigma_{t}\right)$, with covariance matrix $\Sigma_{t}$ given by Eq. (B.5). Thus, ignoring a constant, the logarithm of the likelihood may be written as

$$
\log L_{Y_{N}}=-\frac{1}{2} \sum_{k=1}^{N}\left(\log \left|\Sigma_{k}\right|+\epsilon_{k}^{T} \Sigma_{k}^{-1} \epsilon_{k}\right),
$$

Both criteria have been plotted in Fig. $7\left(\right.$ b) for the simulated data. We see how AIC has a minimum at $n_{s}=16$ and SIC has a minimum at $n_{s}=14$.

- Singular values of the weighted projection matrix in SI [15]. The order of the system (9) is equal to the number of singular values of the weighted projection matrix different from zero. The weighted projection matrix, $W_{1} \mathcal{O}_{i} W_{2}$, is computed using the output block Hankel matrices which are built in the formulation of SSI-data [15]. In Fig. 7(c) we have plotted the singular values for the simulated accelerations; the first 16 singular values are greater than the rest, but there are several singular values different from zero. When using measured data, this method generally does not indicate a clearly defined choice of the model order.

We propose to plot the modal contribution (or the error contribution) and then select the model order from it at the point where the plot becomes constant. This procedure has the followings properties: it is very easy to derive and to implement, it has a physical meaning, the computational cost is low, and it gives good order estimation.

This plot is a good complement for the stabilization diagram, like in Fig. 8, where we have added the modal contribution on the right of the stabilization diagram. The joint analysis of the stabilization diagram and the modal contribution is helpful to better understand the results provided by the identification algorithms. For example, we see that

a

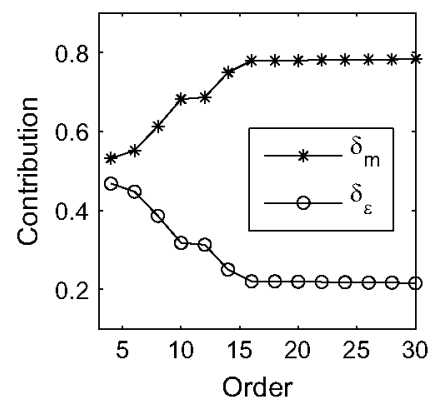

b

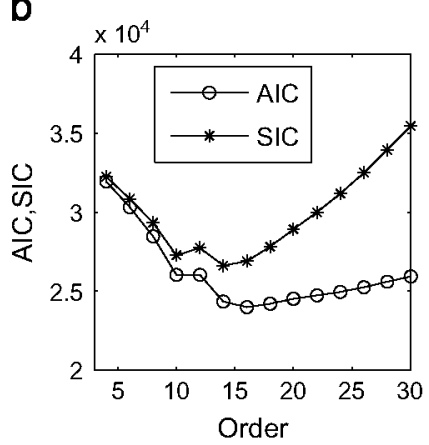

C

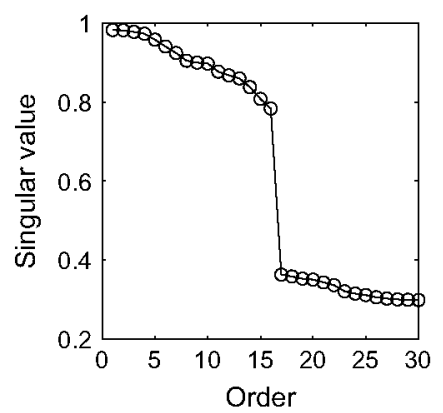

Fig. 7. (a) Modal and error contribution; (b) AIC and SIC criteria; (c) singular values of the weighted projection matrix in SSI. All the plots have been built using the simulated data. 
the modal contribution becomes constant at order $n_{s}=16$, and at this order the identified modes are stable. For higher orders, the extra modes obtained with SSI do not contribute to the signal and are no stable. With the exception of a mode with frequency next to $14.5 \mathrm{~Hz}$. This mode seems to be stable but the modal contribution is negligible, $\delta_{m_{k}}=1.55 \times 10^{-5}$, and the spectrum do not show a peak at this frequency. In fact, this mode has appeared using SSI and the Canonical Variate Algorithm (CVA) with orders higher than the exact; the modes obtained with SSI and the Principal Component algorithm (PC) do not include this mode, but other spurious modes with similar behaviour appear (see [15] for details on the CVA and $\mathrm{PC}$ algorithms). So we should be cautious about modes that are stable in the stabilization diagram but they do not contribute to the measured signal.

\section{Applications: steel transmitter mast}

In this section, the modal contribution and the state space order selection are computed from real measured data. We have chosen a steel frame structure with antennae attached at the top (Fig. 9). This structure has been deeply analysed in [12,13].

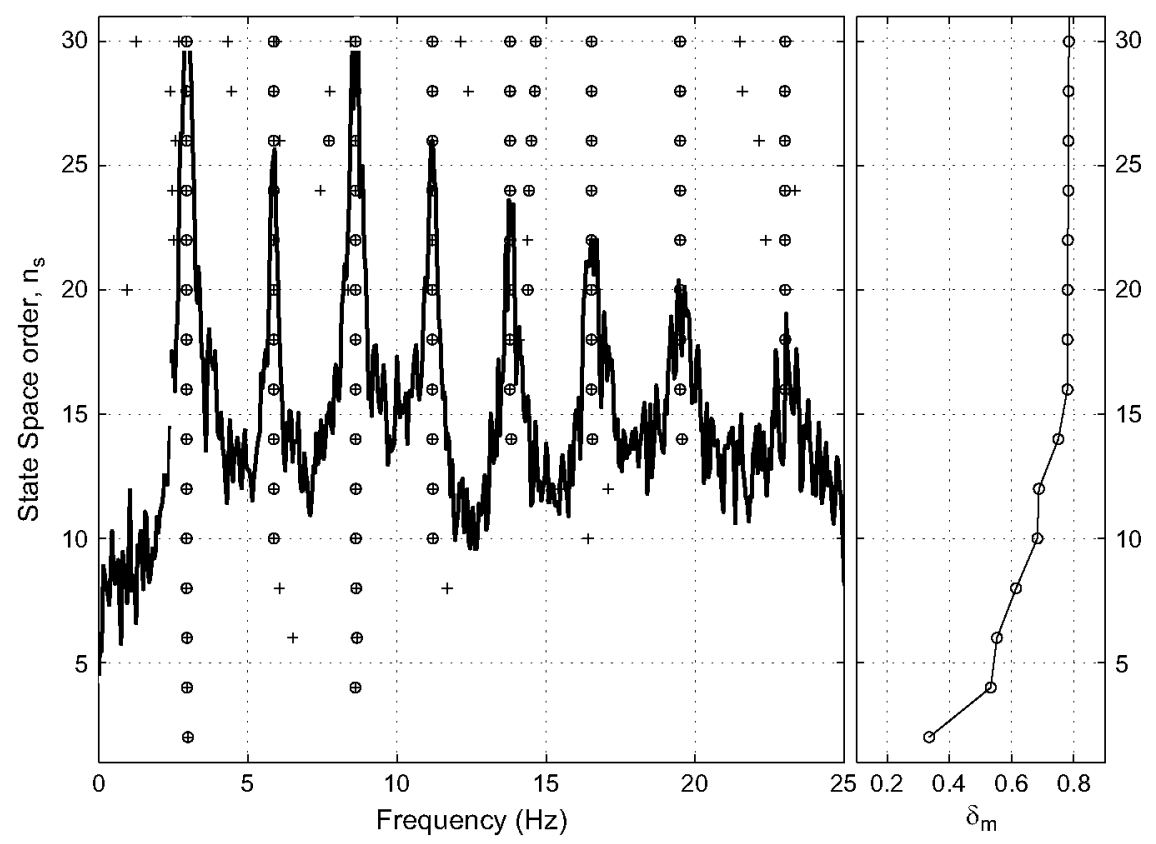

Fig. 8. Left: Stabilization diagram corresponding to the simulated accelerations. The criteria are $2 \%$ for frequencies, $5 \%$ for damping ratios, $5 \%$ for mode shape vectors (MAC). $\oplus$, stable mode; +, unstable mode. The ANPSD is plotted in light gray; Right: modal contribution.

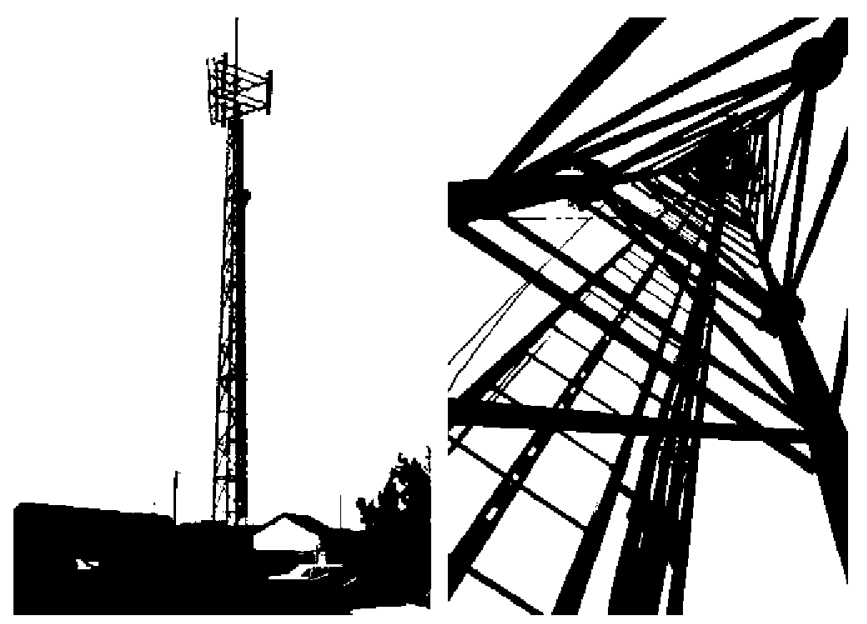

Fig. 9. Steel mast with sectorial antennae at the top. 

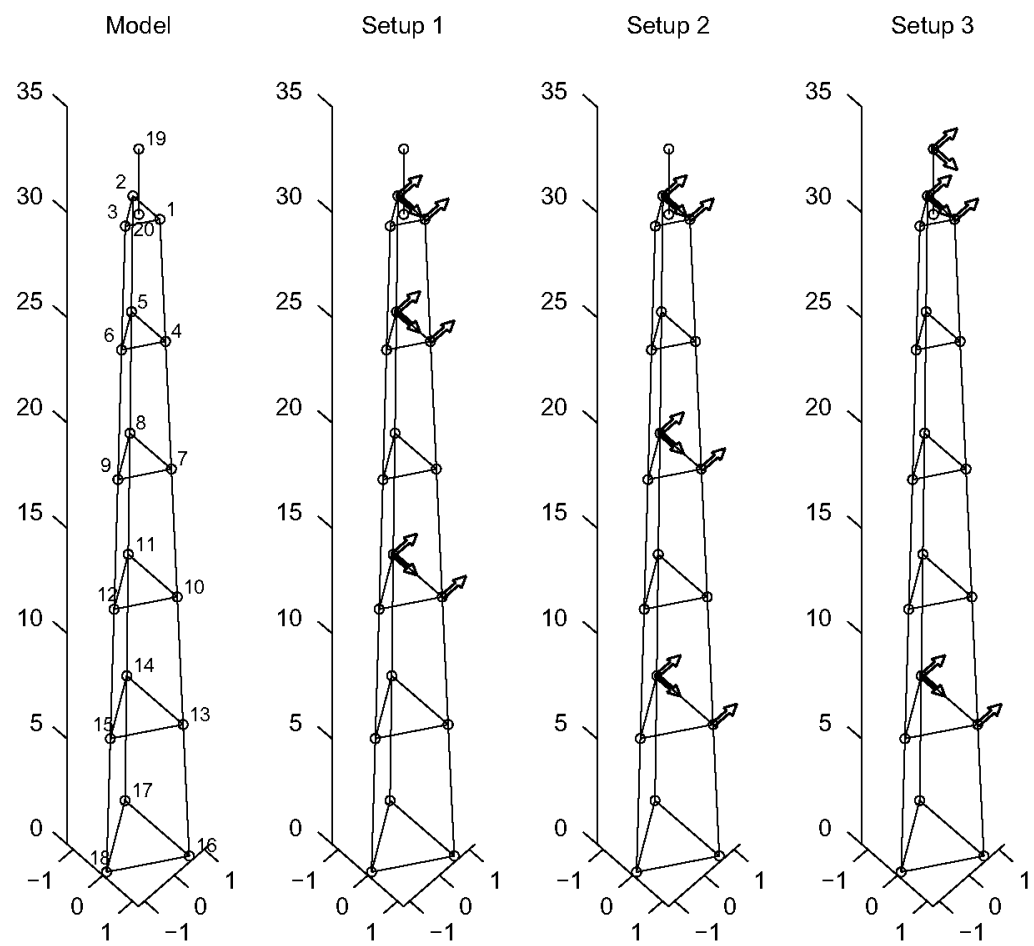

Fig. 10. Steel mast grid and description of the three setups of sensors (the arrows indicate sensor position and measured direction).

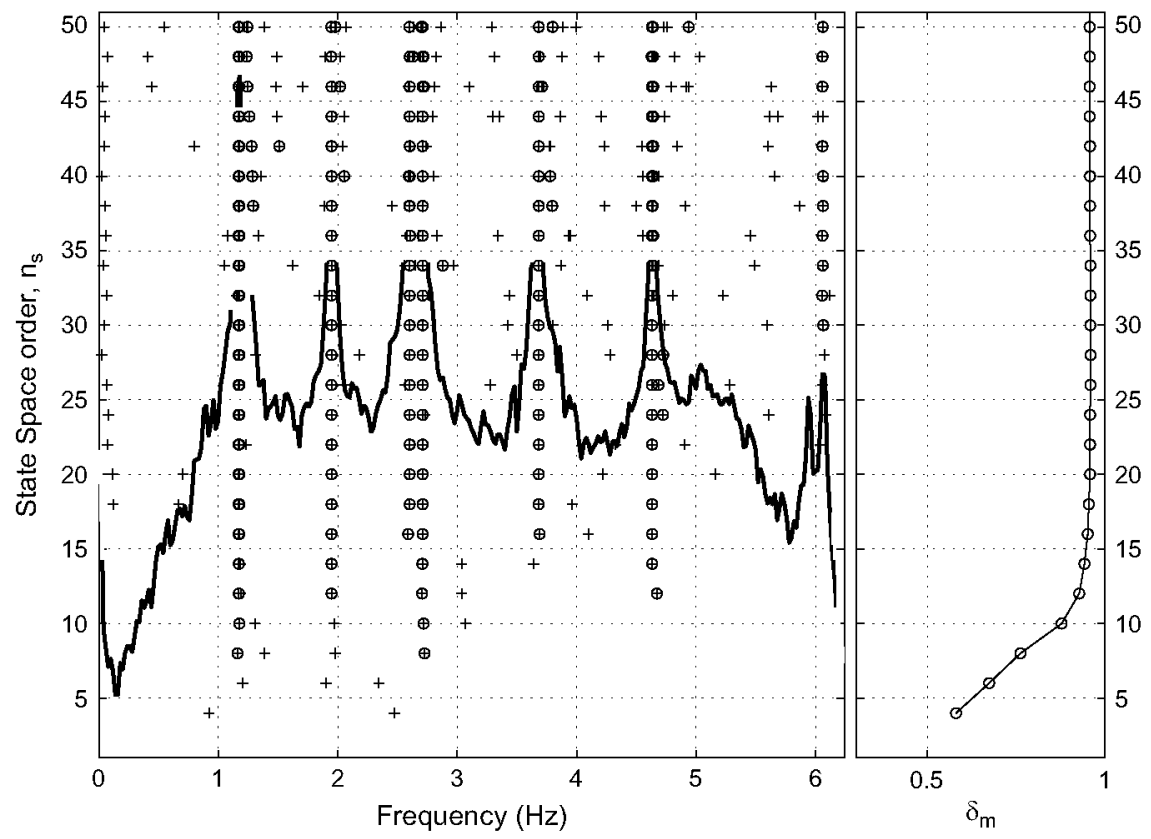

Fig. 11. Stabilization diagram corresponding to the setup 1 . The criteria are $2 \%$ for frequencies, $5 \%$ for damping ratios, $5 \%$ for mode shape vectors (MAC). $\oplus$, stable mode; + , unstable mode. The ANPSD is plotted in light gray.

On March 26, 1998, the structure was subject to ambient vibration measurements. The aim of the test was to investigate the structure's modal damping in the frequency range $0-5 \mathrm{~Hz}$. Seventeen degrees of freedom, all horizontal accelerations, have been measured in three setups using three reference degrees of freedom that were common to each setup (Fig. 10). Three horizontal accelerations have been measured at a height of 6.17, 12.17, 18.17, 24.17 and 29.90 $\mathrm{m}$. The two orthogonal accelerations at the top of the mast (at height of $33.00 \mathrm{~m}$ ) have been measured as well. 
The data were sampled at a rate of $100 \mathrm{~Hz}$. The cut-off frequency of the anti-aliasing filter that was used was set to $20 \mathrm{~Hz}$. The number of samples was set to $N=30720$, which resulted in a measurement time of approximately 5 min. Afterwards, the data were digitally filtered with a low-pass filter with a cut-off frequency of $5 \mathrm{~Hz}$ and resampled at $12.5 \mathrm{~Hz}$, which reduced the number of samples to $N=3840$.

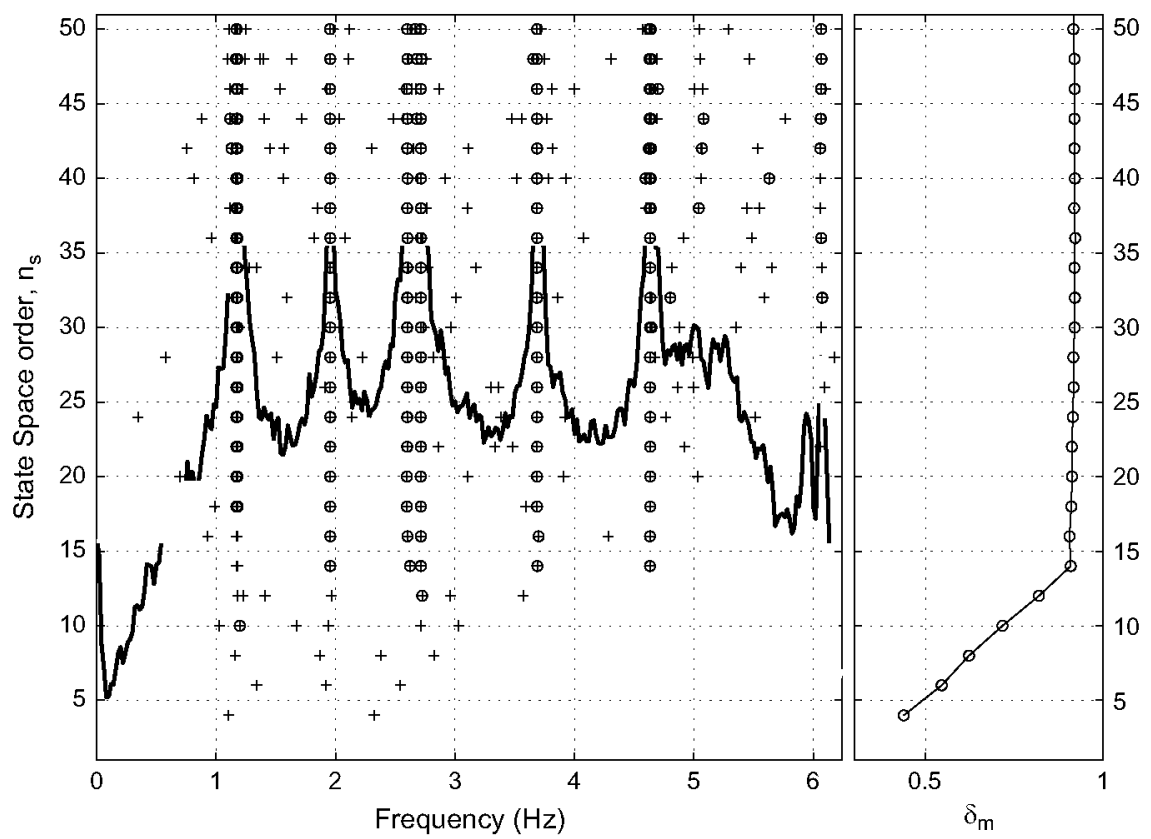

Fig. 12. Stabilization diagram corresponding to the setup 2 . The criteria are $2 \%$ for frequencies, $5 \%$ for damping ratios, $5 \%$ for mode shape vectors (MAC) $\oplus$, stable mode; + , unstable mode. The ANPSD is plotted in light gray.

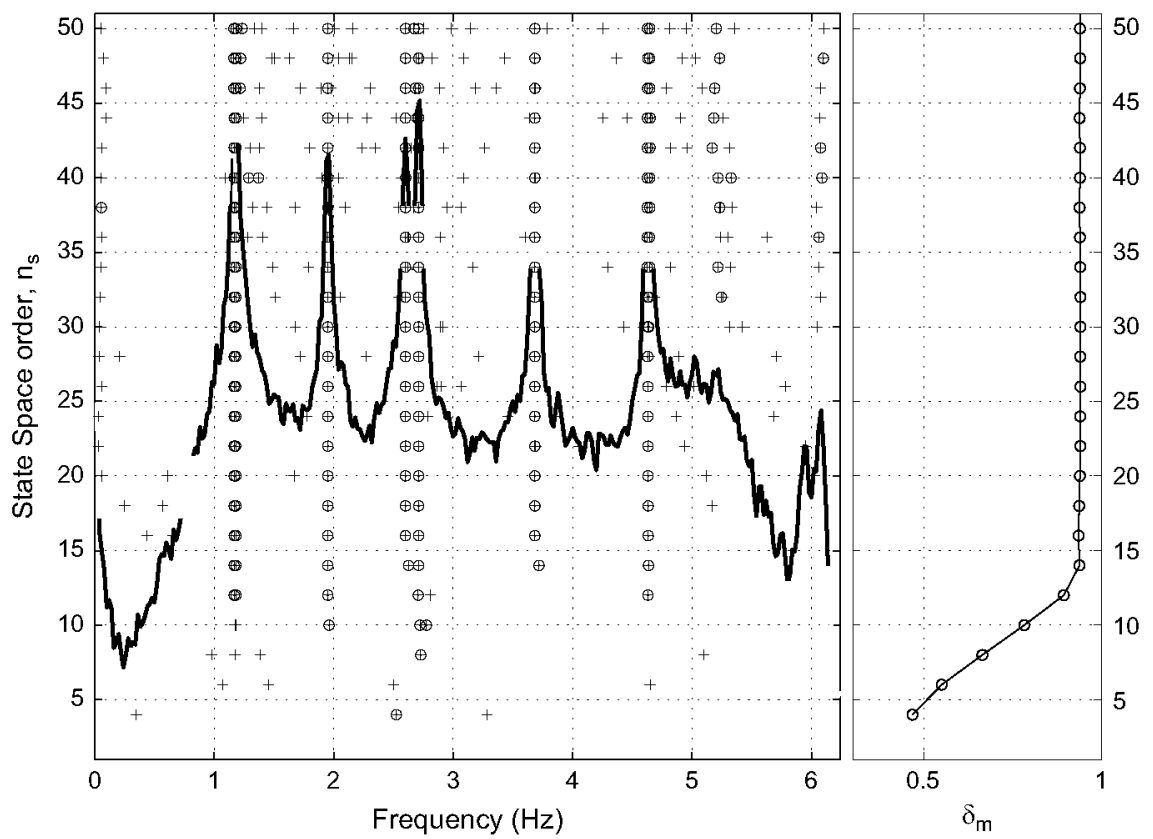

Fig. 13. Stabilization diagram corresponding to the setup 3 . The criteria are $2 \%$ for frequencies, $5 \%$ for damping ratios, $5 \%$ for mode shape vectors (MAC). $\oplus$, stable mode; +, unstable mode. The ANPSD is plotted in light gray. 
We have plotted the stabilizations diagrams for setups 1, 2 and 3 in Figs. 11-13. These stabilization diagrams also include the plot of the modal contribution on the right. We highlight the following aspects:

- In setup 1, the modal contribution increases from 0.58 to around 0.96 . Therefore, the final contribution of the error is 0.04: the mathematical model we have fitted to the data explains $96 \%$ of the measured data.

- The modal contribution becomes approximately constant from $n_{5}=16$, what means that there are eight modes which provide nearly all the modal contribution.

- In setup 2, the modal contribution increases from 0.44 to 0.92 . Therefore, the contribution of the error is 0.08 . The order from which the modal contribution become constant is $n_{s}=14$.

Table 2

Modal contribution by measured DOF, direction and setup.

\begin{tabular}{rllll}
\hline Node & Direct. & Setup 1 & Setup 2 & Setup 3 \\
\hline 1 & $X$ & 0.9622 & 0.9616 & 0.9664 \\
2 & $X$ & 0.9679 & 0.9607 & 0.9683 \\
2 & $Y$ & 0.9797 & 0.9595 & 0.9762 \\
4 & $X$ & 0.9597 & - & - \\
5 & $X$ & 0.9611 & - & - \\
5 & $Y$ & 0.9685 & - & - \\
7 & $X$ & - & 0.9198 & - \\
8 & $X$ & - & 0.9263 & - \\
8 & $Y$ & - & 0.9533 & - \\
10 & $X$ & 0.9375 & - & - \\
11 & $X$ & 0.9089 & - & - \\
11 & $Y$ & 0.9670 & - & - \\
13 & $X$ & - & 0.8338 & 0.8940 \\
14 & $X$ & - & 0.7989 & 0.8432 \\
14 & $Y$ & - & 0.9369 & 0.9450 \\
19 & $X$ & - & - & 0.9603 \\
19 & $Y$ & - & - & 0.9590 \\
& $\delta_{m}$ & 0.9570 & 0.9168 & 0.9390 \\
\hline
\end{tabular}
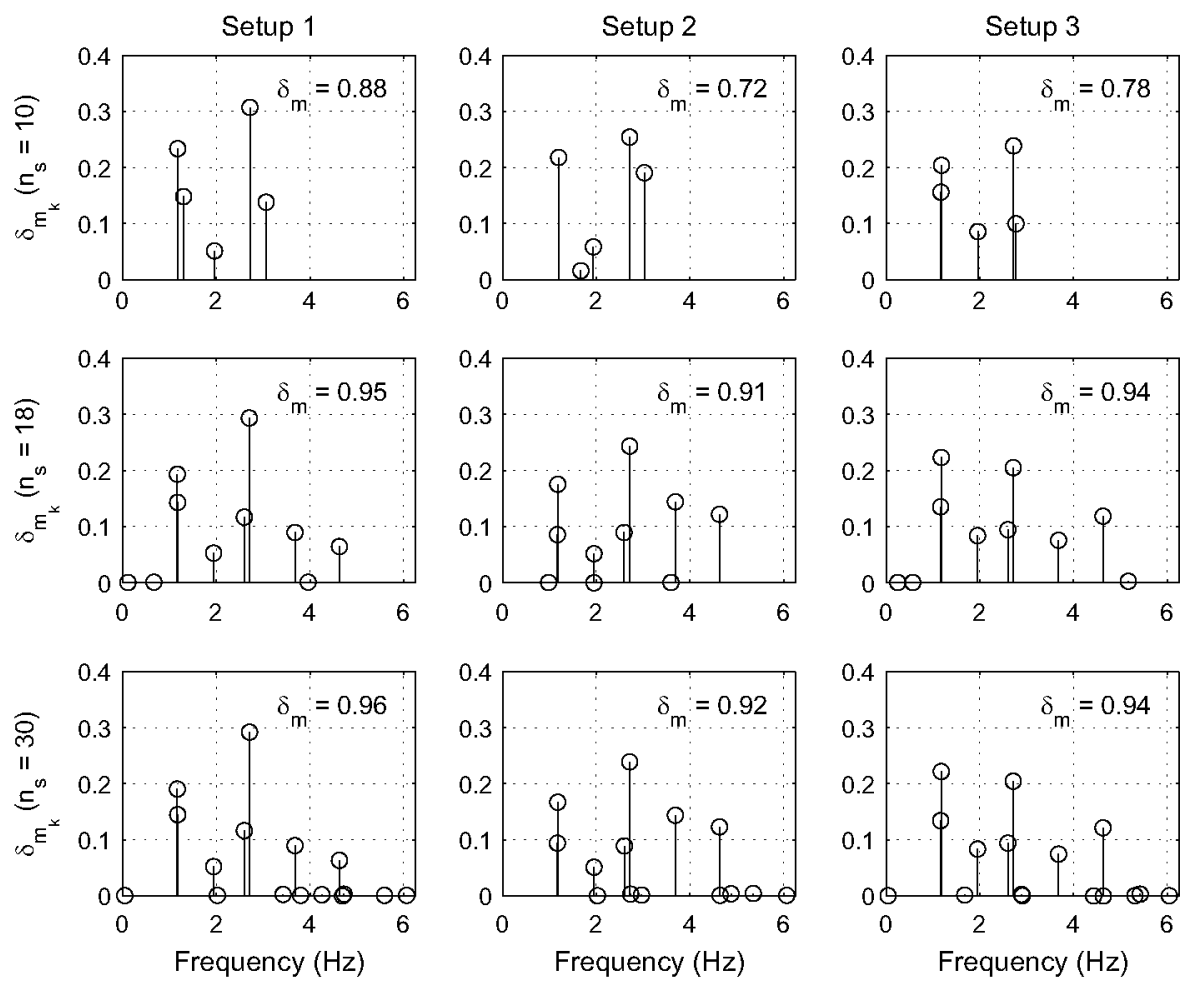

Fig. 14. Modal contribution of the modes identified at model orders $n_{s}=10,18$ and 30 for setups 1,2 and 3 . 
- Setup 2 is a clear example of the interaction between stabilization diagram and the modal contribution plot: observing the latter, we should choose an order equal to 14 . However, at this order the two first modes are not stable at the stabilization diagram, and they do not become stable until order 18 , so this is the model order we chose for setup 2.

- In setup 3, the modal contribution increases from 0.47 to 0.94 , so the error contribution is 0.06 . The order from which the modal contribution become constant is $n_{s}=14$.

- In setup 2, the transition between the increasing values and the constant values is very sharp. However, in setups 1 and 3 this transition is smooth.

- It is interesting to point out that the contribution of the modes to the measured vibration is different for each setup: $0.96,0.92$ and 0.94 , respectively. To understand the differences, we show in Table 2 the contribution of the modes to the vibration measured at each DOF $\left(\delta_{m_{k}}\right)$ :

- The modal contribution at nodes 1 and 2 (the DOF present in the three setup) is high and similar for the three setups.

- Nodes 13 and 14 are also common to setups 2 and 3. The modal contribution is a bit different between setups but the pattern is the same: the modal contribution is higher in the $Y$-direction, and in the $X$-direction, it is higher at node 14.

a

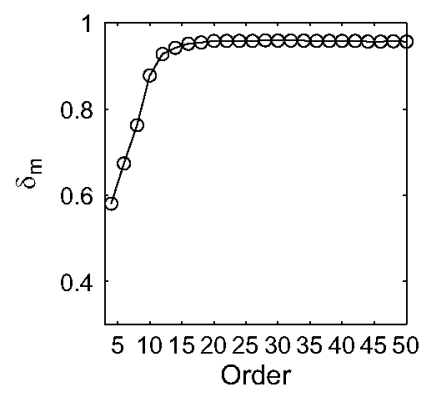

a

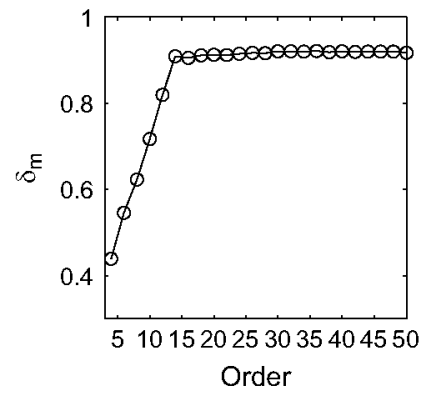

a

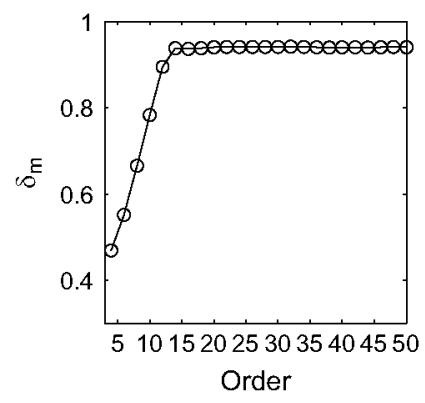

\section{Setup 1}

b

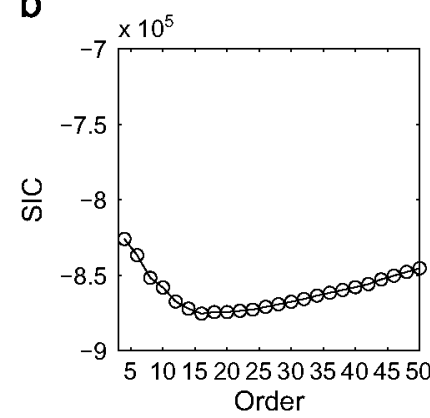

C

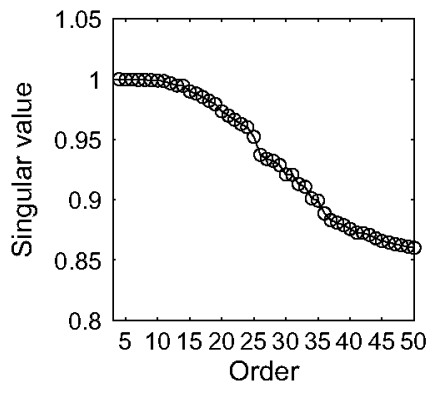

Setup 2

b

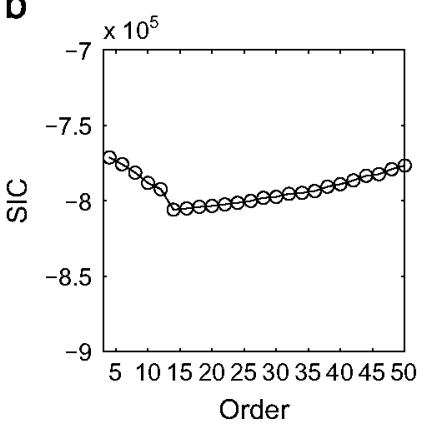

C

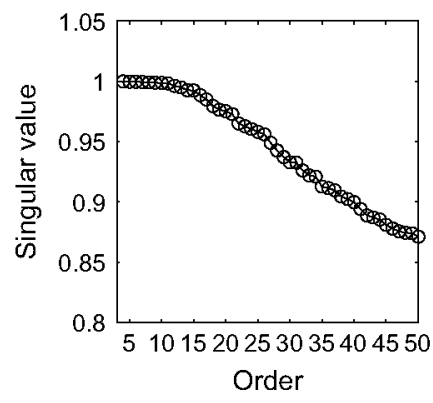

Setup 3

b

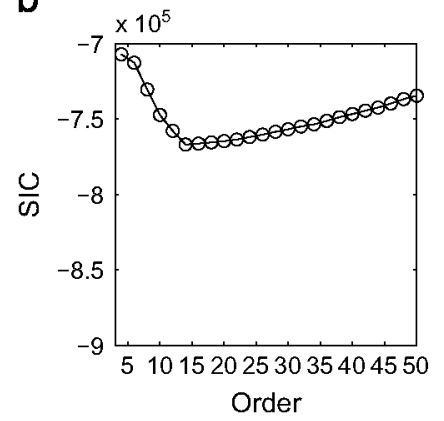

C

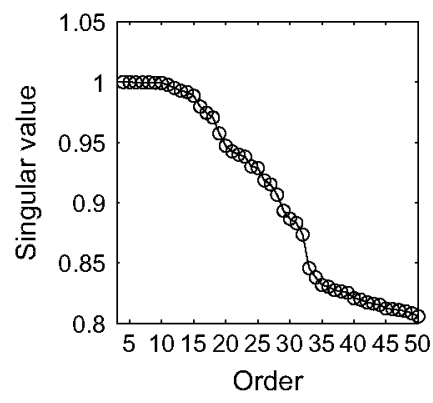

Fig. 15. (a) Modal contribution, $\delta_{m}$; (b) AIC criteria; (c) singular values of the weighted projection matrix in SSI. 

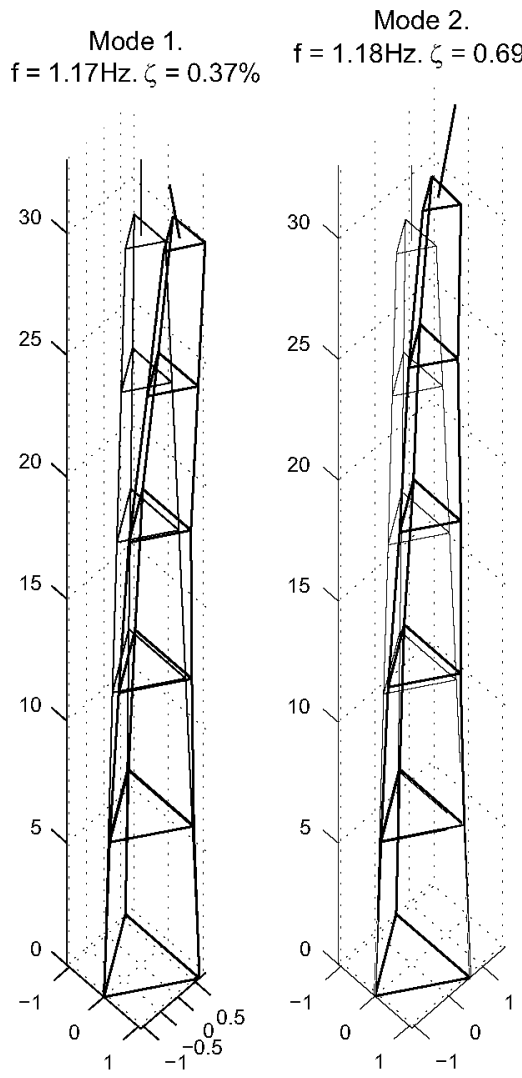

Mode 3.
$f=1.95 \mathrm{~Hz} . \zeta=0.60 \%$

Mode 4.

$f=2.60 \mathrm{~Hz} . \zeta=0.50 \%$

$\begin{array}{ccc}\text { Mode } 5 . & \text { Mode } 6 . & \text { Mode } 7 . \\ f=2.71 \mathrm{~Hz} . \zeta=0.18 \% & f=3.69 \mathrm{~Hz} . \zeta=0.35 \% & f=4.63 \mathrm{~Hz} . \zeta=0.17 \%\end{array}$
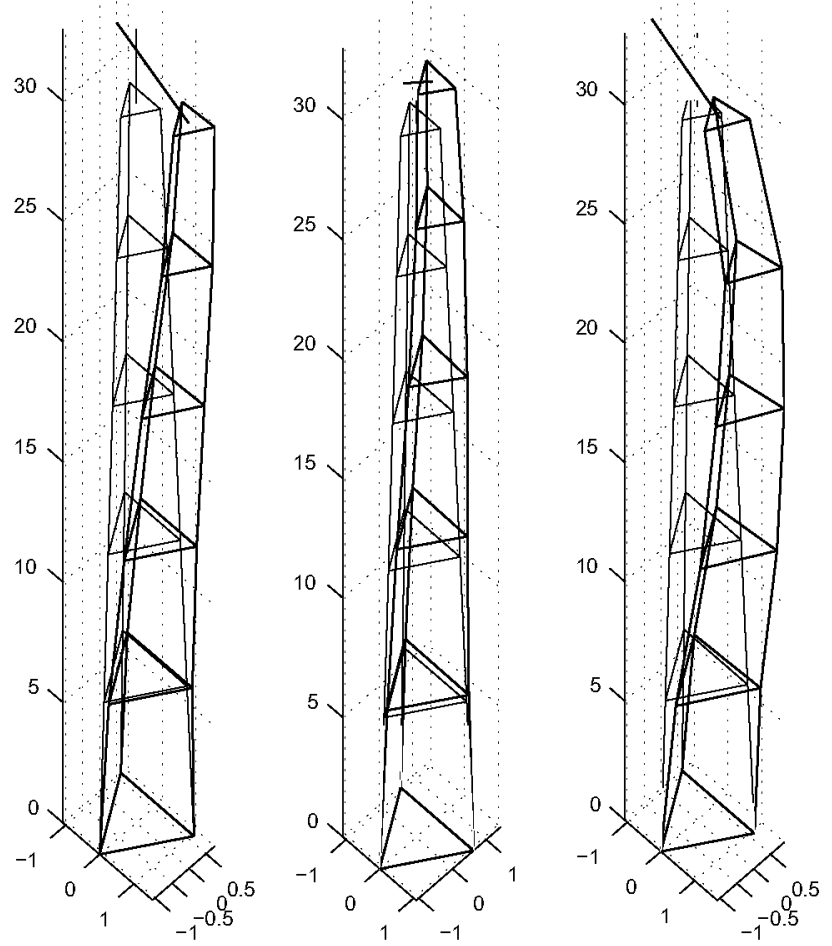

Fig. 16. Identified modes using the optimum state space order: 16,18 and 16 for setups 1,2 and 3 , respectively. The natural frequencies and damping ratios are the mean values of the three setups. 
The vibration measured at nodes located at the top of the steel mast (nodes $1,2,4,5$ and 19 ) is mainly due to the identified modes because the modal contribution is near to one. However, the nodes at the center of the steel mast (noes $7,8,10,11,13$ and 14) have lower modal contribution, so the identified modes do not reproduce so well the vibration at these points.

The Y-direction is better excited because the modal contribution is high at the nodes measured in this direction.

Figs. 14 and 15 are the equivalent to Figs. 5 and 7, but now computed using real measured data, the steel mast data. Fig. 14 shows the modal contribution of the modes identified at model orders $n_{s}=10,18$ and 30 for setups 1,2 and 3 . Taking into account a given setup, the modal contribution of a single mode identified at $n_{s}=10$ can be different than the modal contribution of the same mode identified at $n_{s}=18$ or $n_{s}=30$. However, the modal contribution is the same for $n_{s}=18$ and $n_{s}=30$. This is why the modal contribution of all the modes becomes constant. On the contrary, modes with modal contribution near zero, specially those identified for $n_{s}=30$, correspond to spurious modes in the stabilization diagram. Fig. 14 is important because shows that this also happens taking into account experimental data and different sensor configurations.

With respect to a given model order, the modal contribution of a single mode varies from one setup to the next, but the pattern is the same at the three setups: the mode around $2.7 \mathrm{~Hz}$ has the higher contribution, followed by the ones at $1.2 \mathrm{~Hz}$.

Fig. 15 shows the model order criteria for the steel mast data. The results obtained with the SIC criteria are similar to the ones obtained using the proposed method: $n_{s}=16$ for setup $1, n_{s}=14$ for setup 2 and $n_{5}=14$ for setup 3 . In the case of the singular values of the weighted projection matrix, the selection of a model order is not so straightforward. We observe that there is a change of the singular values behaviour between $n_{s}=10$ and $n_{s}=20$, but this transition is soft and it is not easy to derive the system order.

Finally, Fig. 16 shows the modes identified from the measured data. We have compared all the modal parameters estimated at $n_{s}=16$ in setup 1 , at $n_{s}=18$ in setup 2 and at $n_{s}=16$ in setup $3:$ mode $i$ and mode $j$ estimated at setup $p$ and setup $q$, respectively, correspond to the same structural mode, if they verify

$$
\begin{aligned}
& \frac{\left|f_{p i}-f_{q j}\right|}{f_{p i}} \leq 0.05, \\
& \frac{\left|\zeta_{p i}-\zeta_{q j}\right|}{\zeta_{p i}} \leq 0.05, \\
& 1-M A C\left(\phi_{p i}, \phi_{q j}\right) \leq 0.10,
\end{aligned}
$$

(the MAC values have been computed using only the reference sensors). It is important to note that, given the chosen order for each setup, we can automatize this procedure of gluing mdes. That is what we have done in Fig. 16 .

The natural frequencies and damping ratios included in the figure are the mean value of the modal parameters estimated in the three setups.

The modes of the steel mast have been previously estimated in [12,13]. The modes obtained in this work using the selected orders for each setup (Fig. 16), match quite well the ones presented in those works.

\section{Conclusions}

In this paper we have presented some post-processing tools for operational modal analysis. First, we have estimated the vibration response corresponding to each identified mode by mean of the Kalman filter. Then, we have developed a procedure to quantify the contribution of this modal vibration to the measured vibration. Two important aspects related to this procedure were investigated: the relative importance of the estimated modes and the order of the state space equations.

In operational modal analysis it is common to estimate the modal parameters at different state space orders and then to construct the stabilization diagram. We have proposed to compute the modal contribution at the same orders used in the stabilization diagram, and then to plot the modal contribution next to the stabilization diagram. The joint analysis of both plots is very useful for modal estimation and order selection. For example, we have found that the modal contribution becomes constant from a certain order, which means that at this order we have estimated the modes with the highest modal contribution. For this reason we have proposed to estimate the modal parameters of the structure at this order.

On the other hand, the modal contribution and the selected order can be used for gluing the modes estimated at different configurations of sensors. The point is that, once the order is selected for each configuration, we can glue automatically the modes. Moreover, it would be even possible to select the state space order automatically, so the whole process could be performed without user help. 
The accuracy and practicability of the techniques have been illustrated with representative simulation and real-life vibration data.

\section{Acknowledgments}

This research was supported by the Ayudas del Consejo Social de la Universidad Politécnica de Madrid a la Formación de Doctorandos, curso 2010-2011. The financial support is gratefully acknowledged.

\section{Appendix A. Nomenclature}

\begin{tabular}{|c|c|c|c|}
\hline Symbol & Description & & Defined at Eq. \\
\hline$A_{\mathrm{C}}, A$ & transition state matrix in continuous and discrete time & $\mathbb{R}^{n_{5} \times n_{5}}$ & $(3),(10)$ \\
\hline$B_{c}, B$ & input matrix in continuous and discrete time & $\mathbb{R}^{n_{5} \times n_{i}}$ & $(3),(10)$ \\
\hline$C_{a}$ & measurement location matrix for accelerations & $\mathbb{R}^{n_{0} \times n_{5}}$ & (4) \\
\hline$C_{c}, C$ & output matrix in continuous and discrete time & $\mathbb{R}^{n_{0} \times n_{5}}$ & $(6),(10)$ \\
\hline$D$ & direct transmission matrix in continuous and discrete time & $\mathbb{R}^{n_{0} \times n_{i}}$ & $(6),(10)$ \\
\hline $\mathcal{D}$ & damping matrix & $\mathbb{R}^{n_{d} \times n_{d}}$ & (1) \\
\hline$J$ & excitation influence matrix & $\mathbb{R}^{n_{5} \times n_{i}}$ & (1) \\
\hline$K$ & Kalman gain & $\mathbb{R}^{n_{5} \times n_{0}}$ & (B.4) \\
\hline $\mathcal{K}$ & stiffness matrix & $\mathbb{R}^{n_{\mathrm{d}} \times n_{\mathrm{d}}}$ & (1) \\
\hline $\mathcal{M}$ & mass matrix & $\mathbb{R}^{n_{d} \times n_{d}}$ & (1) \\
\hline $\operatorname{MAC}\left(v_{i}, v_{j}\right)$ & Modal assurance criterion between vector $v_{i}$ and vector $v_{j}$ & {$[0,1]$} & $(22)$ \\
\hline$Q$ & variance matrix of $w_{k}$ & $\mathbb{R}^{n_{5} \times n_{5}}$ & $(12)$ \\
\hline$R$ & variance matrix of $v_{k}$ & $\mathbb{R}^{n_{0} \times n_{0}}$ & $(12)$ \\
\hline$S$ & covariance matrix between $w_{k}$ and $v_{k}$ & $\mathbb{R}^{n_{5} \times n_{0}}$ & $(12)$ \\
\hline$f_{s}$ & sampling frequency $(\mathrm{Hz})$ & $\mathbb{R}_{+}$ & \\
\hline$n_{i}$ & number of degrees of freedom & $\mathbb{N}$ & \\
\hline$n_{\mathrm{i}}$ & number of inputs in the state space equations & $\mathbb{N}$ & \\
\hline$n_{0}$ & number of outputs in the state space equations & $\mathbb{N}$ & \\
\hline$n_{S}$ & number of states in the state space equations & $\mathbb{N}$ & \\
\hline$q(t)$ & $n_{d}$-dimensional displacement response vector & $\mathbb{R}^{n_{d} \times 1}$ & (1) \\
\hline$u(t), u_{k}$ & input vector in continuous and discrete time & $\mathbb{R}^{n_{i} \times 1}$ & (1) \\
\hline$v_{k}$ & measurement noise vector & $\mathbb{R}^{n_{0} \times 1}$ & (11) \\
\hline$w_{k}$ & state noise vector & $\mathbb{R}^{n_{5} \times 1}$ & (11) \\
\hline$x(t), x_{k}$ & state vector in continuous and discrete time & $\mathbb{R}^{n_{5} \times 1}$ & $(7),(9)$ \\
\hline$x_{k \mid k-1}$ & expected value of $x_{k}$ given the output measurements $\left\{y_{1}, y_{2}, \ldots, y_{k-1}\right\}$ & $\mathbb{R}^{n_{5} \times 1}$ & \\
\hline$y(t), y_{k}$ & output vector in continuous and discrete time & $\mathbb{R}^{n_{0} \times 1}$ & $(7),(9)$ \\
\hline$y_{k}^{m}$ & output vector due to the identified modes & $\mathbb{R}^{n_{0} \times 1}$ & (29) \\
\hline$y_{k}^{\epsilon}$ & output vector due to error & $\mathbb{R}^{n_{0} \times 1}$ & (29) \\
\hline$\Delta_{m}(n)$ & contribution of the identified modes to the measured vibration at channel $n$ & {$[0,1]$} & (34) \\
\hline$\Delta_{m_{k}}(n)$ & contribution of the identified mode $k$ to the measured vibration at channel $n$ & {$[0,1]$} & (36) \\
\hline$\Delta t$ & sampling period (s) & $\mathbb{R}_{+}$ & \\
\hline$\Delta_{\epsilon}(n)$ & contribution of the error to the measured vibration at channel $n$ & {$[0,1]$} & (34) \\
\hline$\delta_{m}$ & global contribution of the modes & {$[0,1]$} & (35) \\
\hline$\delta_{m_{k}}$ & global contribution of mode $k$ & {$[0,1]$} & (37) \\
\hline$\delta_{E}$ & global contribution of the error & {$[0,1]$} & (35) \\
\hline$\epsilon_{k}$ & innovations & $\mathbb{R}^{n_{0} \times 1}$ & (B.3) \\
\hline$\varepsilon_{\gamma}, \varepsilon_{\zeta}, \varepsilon_{M A C}$ & tolerance limits for frequency, damping ratio and $\mathrm{MAC}$, respectively & $\mathbb{R}_{+}$ & $(19)-(21)$ \\
\hline$\lambda_{j}$ & eigenvalue $j$ of matrix $A$ & $\mathbb{C}$ & (15) \\
\hline$\omega_{j}$ & natural frequency of mode $j(\mathrm{rad} / \mathrm{s})$ & $\mathbb{R}_{+}$ & $(16)$ \\
\hline$\zeta_{j}$ & damping ratio of mode $j$ & {$[0,1]$} & (17) \\
\hline$\phi_{j}$ & jth mode shape evaluated at sensor locations & $\mathbb{R}^{n_{0} \times 1}$ & (18) \\
\hline$\psi_{j}$ & eigenvector of matrix $A$ corresponding to eigenvalue $\lambda_{j}$ & $\mathbb{C}^{n_{5} \times 1}$ & \\
\hline
\end{tabular}

\section{Appendix B. The Kalman filter}

The following notation has been used in all the expressions where the Kalman filter is involved. Given the output data for $s$ time steps $Y_{s}=\left\{y_{1}, y_{2}, \ldots, y_{s}\right\}$, it is defined:

$$
\begin{aligned}
& x_{t \mid s}=\mathrm{E}\left[x_{t} \mid Y_{s}\right] \\
& P_{t_{1}, t_{2} \mid s}=\mathrm{E}\left[\left(x_{t_{1}}-x_{t_{1} \mid s}\right)\left(x_{t_{2}}-x_{t_{2} \mid s}\right)^{T} \mid Y_{s}\right]
\end{aligned}
$$


where $\mathrm{E}[\bullet \mid \bullet]$ is the conditional expectation operator. When $t_{1}=t_{2}=t, P_{t_{1}, t_{2} \mid s}$ will be written $P_{t \mid s}$ :

$$
P_{t \mid s}=\mathrm{E}\left[\left(x_{t}-x_{t \mid s}\right)\left(x_{t}-x_{t \mid s}\right)^{T} \mid Y_{s}\right]=\operatorname{Var}\left[x_{t} \mid Y_{s}\right]
$$

Property 1 (The Kalman Filter). For the state space model specified in (13) $x_{1 \mid 0}$ and $P_{1 \mid 0}$, for $k=1,2, \ldots, N$,

$$
\begin{aligned}
& x_{k+1 \mid k}=A x_{k \mid k-1}+K_{k} \epsilon_{k} \\
& P_{k+1 \mid k}=A P_{k \mid k-1} A^{T}+Q-K_{k} \Sigma_{k} K_{k}^{T}
\end{aligned}
$$

where

$$
\begin{aligned}
& \epsilon_{k}=y_{k}-C x_{k \mid k-1} \\
& K_{k}=\left(A P_{k \mid k-1} C^{T}+S\right) \Sigma_{k}^{-1} \\
& \Sigma_{k}=\operatorname{Var}\left(\epsilon_{k}\right)=C P_{k \mid k-1} C^{T}+R
\end{aligned}
$$

$K_{k}$ is called the Kalman gain and $\epsilon_{k}$ are denominated the innovations. The filter values are given by

$$
\begin{aligned}
& x_{k \mid k}=x_{k \mid k-1}+P_{k \mid k-1} C^{T}\left(C P_{k \mid k-1} C^{T}+R\right)^{-1} \epsilon_{k+1} \\
& P_{k \mid k}=P_{k \mid k-1}-P_{k \mid k-1} C^{T}\left(C P_{k \mid k-1} C^{T}+R\right)^{-1} C P_{k \mid k-1}
\end{aligned}
$$

The demonstration of the above property can be found, for example, in [17]. Under stationary conditions (see for instance [19]),

$$
\lim _{k \rightarrow \infty} P_{k \mid k-1}=P>0
$$

where

$$
\begin{aligned}
& P=A P A^{T}+Q-\left(A P C^{T}+S\right)\left(C P C^{T}+R\right)^{-1}\left(A P C^{T}+S\right)^{T} \\
& K=\left(A P C^{T}+S\right)\left(C P C^{T}+R\right)^{-1}
\end{aligned}
$$

Eq. (B.9) is called a discrete algebraic Riccati equation (DARE) and it is a steady-state version of Eq. (B.2). These stationary conditions are satisfied in linear time invariant (LTI) systems.

\section{References}

[1] A. Pavic, Z. Miskovic, P. Reynolds, Modal testing and FE model updating of a lively open-plan composite building floor, J. Struct. Eng. ASCE 133 (4) (2007) $550-558$.

[2] E. Reynders, A. Teughels, G. De Roeck, Finite element model updating and structural damage identification using OMAX data, Mech. Syst. Signal Process. 24 (5) (2010) 1306-1323.

[3] S.W. Doebling, C.R. Farrar, M.B. Prime, D.W. Shevitz, Damage Identification and Health Monitoring of Structural and Mechanical Systems from Changes in Their Vibrational Characteristics: A literature Review, Technical Report LA-13070-MS, Los Alamos National Laboratory, 1996.

[4] E. Reynders, G. De Roeck, A local flexibility method for vibration-based damage localization and quantification, J. Sound Vib. 329 (12) (2010) $2367-2383$.

[5] E. Parloo, P. Verboven, P. Guillaume, M. Van Overmeire, Force identification by means of in-operation modal models, J. Sound Vib. 262 (1) (2003) $161-173$.

[6] E. Lourens, E. Reynders, G. De Roeck, G. Degrande, G. Lombaert, An augmented Kalman filter for force identification in structural dynamics, Mech. Syst. Signal Process. 27 (2012) 446-460.

[7] B. Peeters, System Identification and Damage Detection in Civil Engineering. Ph.D. Thesis, Katholieke Universiteit Leuven, 2000.

[8] B. Alicioglu, M. Lus, Ambient vibration analysis with subspace methods and automated mode selection: case studies, J. Struct. Eng. ASCE. (June) (2008) 1016-1029.

[9] H. Van der Auweraer, B. Peeters, Discriminating Physical Poles from Mathematical Poles in High Order Systems: Use and Automation of the Stabilization Diagram, IMIC 2004 - Instrumentation and Measurement Technology Conference, Como, Italy, 18-20 May 2004.

[10] M. Scionti, J.P. Lanslots, Stabilisation diagrams: Pole identification using fuzzy clustering techniques, Adv. Eng. Software 36 (11-12, NovemberDecember) (2005) 768-779.

[11] P. Verboven, E. Parloo, P. Guillaume, M. Van Overmeire, Autonomous structural health monitoring - Part I: modal parameter estimation and tracking, Mech. Syst. Signal Process. 16 (July (4)) (2002) 637-657.

[12] B. Peeters, G. De Roeck, Reference-based stochastic subspace identification for output-only modal analysis, Mech. Syst. Signal Process. 13 (6) (1999) $855-878$.

[13] E. Reynders, R. Pintelon, G. De Roeck, Uncertainty bounds on modal parameters obtained from stochastic subspace identification, Mech. Syst. Signal Process. 22 (2008) 948-969.

[14] H. Akaike, Stochastic theory of minimal realization, IEEE Trans. Autom. Control 16 (6) (1974) 667-674.

[15] P. Van Overschee, B. De Moor, Subspace Identification for Linear Systems. Theory - Implementation - Applications, Kluwer Academic Publishers, 1996.

[16] J. Juang, Applied System Identification, Prentice Hall PTR, 1994. 
[17] R.H. Shumway, D.S. Stoffer, Time Series Analysis and its Applications, Springer, 2006.

[18] F.J. Cara, J. Carpio, J. Juan, E. Alarcón, An approach to operational modal analysis using the expectation maximization algorithm, Mech. Syst. Signal Process. 31 (August) (2012) 109-129.

[19] M. Verhaegen, V. Verdult, Filtering and System Identification. A Least Squares Approach, Cambridge University Press, 2007.

[20] E. Reynders, System identification methods for (operational) modal analysis: review and comparison, Arch. Comput. Methods Eng. 19 (2012) 51-124.

[21] L. Ljung, System Identification Theory for the Users, 2nd Ed. PTR Prentice-Hall, Upper Saddle River, NJ, 1999.

[22] E. Reynders, J. Houbrechts, G. De Roeck, Fully automated (operational) modal analysis, Mech. Syst. Signal Process. 29 (May) (2012) $228-250$.

[23] H. Akaike, A new look at the statistical identification model, IEEE Trans. Autom. Control (1974) 716-723.

[24] G. Schwarz, Estimating the dimension of a model, Ann. Stat. 6 (1978) 461-464.

[25] A.B. Koehler, E.S. Murphree, A comparison the Akaike and Schwarz of criteria selecting model order, J. R. Stat. Soc. Ser. C: Appl. Stat. 37 (2) (1988) 187-195. 